\title{
Loss of an H3K9me anchor rescues laminopathy-linked changes in nuclear organization and muscle function in an Emery-Dreifuss muscular dystrophy model
}

\author{
Jennifer C. Harr, ${ }^{1,4}$ Christoph D. Schmid, ${ }^{1}$ Celia Muñoz-Jiménez, ${ }^{2}$ Raquel Romero-Bueno, ${ }^{2}$ \\ Véronique Kalck, ${ }^{1}$ Adriana Gonzalez-Sandoval, ${ }^{1,5}$ Michael H. Hauer, ${ }^{1}$ Jan Padeken, ${ }^{1}$ Peter Askjaer, ${ }^{2}$ \\ Anna Mattout, ${ }^{1,6}$ and Susan M. Gasser ${ }^{1,3}$ \\ ${ }^{1}$ Friedrich Miescher Institute for Biomedical Research, CH-4058 Basel, Switzerland; ${ }^{2}$ Andalusian Center for Developmental \\ Biology (CABD), Consejo Superior de Investigaciones Científicas, Universidad Pablo de Olavide, Seville 41013, Spain; ${ }^{3}$ University \\ of Basel, Faculty of Natural Sciences, CH-4056 Basel, Switzerland
}

Mutations in the nuclear structural protein lamin A produce rare, tissue-specific diseases called laminopathies. The introduction of a human Emery-Dreifuss muscular dystrophy (EDMD)-inducing mutation into the C. elegans lamin (LMN-Y59C), recapitulates many muscular dystrophy phenotypes, and correlates with hyper-sequestration of a heterochromatic array at the nuclear periphery in muscle cells. Using muscle-specific emerin Dam-ID in worms, we monitored the effects of the mutation on endogenous chromatin. An increased contact with the nuclear periphery along chromosome arms, and an enhanced release of chromosomal centers, coincided with the disease phenotypes of reduced locomotion and compromised sarcomere integrity. The coupling of the LMN-Y59C mutation with the ablation of CEC-4, a chromodomain protein that anchors H3K9-methylated chromatin at the nuclear envelope (NE), suppressed the muscle-associated disease phenotypes. Deletion of cec-4 also rescued LMN-Y59C-linked alterations in chromatin organization and some changes in transcription. Sequences that changed position in the LMN-Y59C mutant, are enriched for E2F (EFL-2)-binding sites, consistent with previous studies suggesting that altered Rb-E2F interaction with lamin A may contribute to muscle dysfunction. In summary, we were able to counteract the dominant muscle-specific defects provoked by LMNA mutation by the ablation of a lamin-associated H3K9me anchor, suggesting a novel therapeutic pathway for EDMD.

[Keywords: laminopathy; CEC-4; lamin A; C. elegans; EDMD; nuclear organization; LADs]

Supplemental material is available for this article.

Received August 30, 2019; revised version accepted February 14, 2020.

Laminopathies represent a large set of rare genetic diseases that lead to differentiated tissue dysfunction, arising from mutations in components of the nuclear lamina, the most frequent being lamin A (LMNA). Point mutations in $L M N A$, or in other lamin-associated proteins such as emerin (Worman and Bonne 2007), are linked to $\sim 15$ distinct diseases, many of which lead to premature death by cardiac dysfunction, coupled with striated muscular dystrophy, peripheral neuropathy or, more rarely, lipodystrophy (Worman and Bonne 2007). The majority of the $\sim 450$ mapped laminopathy mutations are autoso-

Present Addresses: ${ }^{4}$ St. Mary's University, San Antonio, Texas 78228, USA; ${ }^{5}$ Stanford University School of Medicine, CA 94305, USA; ${ }^{6}$ UMR 5088, CNRS, Université Paul Sabatier, 31062 Toulouse, France.

Corresponding authors: susan.gasser@fmi.ch, anna.mattout@univ-tlse3.fr Article published online ahead of print. Article and publication date are online at http://www.genesdev.org/cgi/doi/10.1101/gad.332213.119. Freely available online through the Genes \& Development Open Access option. mal dominant missense alleles of LMNA. However, because the mutations occur throughout the gene, without clustering in any one structural domain of the $70-\mathrm{kDa}$ lamin, it has been difficult to link the pathologies to a specific function (Dittmer and Misteli 2011). As a result, there are to date no effective treatments or cures for laminopathic disease.

There are two major hypotheses put forth to explain these diseases, one that implicates structural defects in nuclear organization and a second that implicates lamin A directly in gene regulation (Camozzi et al. 2014; Osmanagic-Myers et al. 2015). These mechanisms are not mutually exclusive, but imply different roles for lamin A. The first argues that lamins, which create a semirigid structure adjacent to the inner nuclear membrane

(C) 2020 Harr et al. This article, published in Genes \& Development, is available under a Creative Commons License (Attribution-NonCommercial 4.0 International), as described at http://creativecommons.org/licenses/by-nc/4.0/. 
(INM), protect the genome from mechanical stress (Worman and Bonne 2007; Dittmer and Misteli 2011). It is proposed that the compromised scaffold alters chromosomal organization and indirectly compromises tissue function, particularly in muscle, which is subject to stretch and compression. Exactly how mechanical stress changes genome function is unclear. The second hypothesis argues that LMNA mutations themselves provoke changes in gene expression, for instance, by altering lamin A interaction with transcription factors that are needed for maintenance of tissue integrity (Lloyd et al. 2002; Mounkes et al. 2005). Evidence for the former rests on studies showing that lamin A contributes to nuclear shape and the spatial distribution of heterochromatin (Solovei et al. 2013; Cho et al. 2019), while the second is based on the observation that lamins bind a range of transcriptional regulators and/or histone variants (Vlcek and Foisner 2007). These latter interactions involve nucleoplasmic lamin A, as well as the peripheral lamin fibers. Very few experiments address rigorously the relevance of either hypothesis. Indeed, defining the molecular pathways that link lamin A to muscle pathology has been difficult due to the multiplicity of $L M N A$ mutations, the complexity of its interactors, and the fact that mouse models are only moderately successful at recapitulating these diseases (Mounkes et al. 2005; Stewart et al. 2007).

Because laminopathies manifest tissue-specific defects that are often late onset (i.e., manifest in adolescence), the use of model organisms to elucidate disease mechanisms is needed. The nematode Caenorhabditis elegans has proven to be a propitious choice (Wiesel et al. 2008; Mattout et al. 2011; Bank and Gruenbaum 2011a), because worms have only one lamin (LMN-1) and lack adult stem cells in somatic tissues, helping us differentiate reduced muscle function from impaired regeneration. Moreover, we and others have amply demonstrated that condensed, transcriptionally silent heterochromatin is found adjacent to the INM in contact with the nuclear lamina in worms as in other metazoans (Ikegami et al. 2010; Meister et al. 2010b; Towbin et al. 2012). The peripheral tethering of chromatin depends, at least in part, on the interaction of heterochromatin-specific histone modifications with INM components (Harr et al. 2016; Cohen-Fix and Askjaer 2017). In C. elegans embryos this is mediated by CEC-4, a nuclear envelope (NE)-associated protein that has a chromodomain (CD) with $46 \%$ identity to the CD of human heterochromatin protein $1 \alpha$ (HP1a) (Gonzalez-Sandoval et al. 2015). CEC-4 specifically tethers nucleosomes bearing histone H3 Lys9 methylation (H3K9me). This mark is essential for peripheral heterochromatin tethering in embryonic nuclei (Towbin et al. 2012; Gonzalez-Sandoval et al. 2015). Intriguingly, after embryonic development a second anchoring pathway is induced, which can be disrupted in L1 stage larvae by ablation of the H3K36me reader, MRG-1, and the release of p300/CBP1 from active chromatin (Cabianca et al. 2019). In vertebrate species, the exact counterparts of these two anchoring pathways remain to be defined, yet two partially redundant pathways that are compromised by ablation of lamin A and lamin B receptor (LBR), respectively, are necessary to maintain heterochromatin at the INM in differentiated cells (Solovei et al. 2013). These also control the expression of genes in early myoblast differentiation (Favreau et al. 2004; Frock et al. 2006; Solovei et al. 2013). An unstructured protein that binds HP1a, called PRR14, may also be involved in both chromatin organization and myoblast differentiation (Poleshko et al. 2013).

We studied the laminopathic mutation (LMNA-Y45C), which results in a degenerative condition in striated muscle called Emery-Dreifuss muscular dystrophy (EDMD). The equivalent mutation was introduced into the worm lamin (LMN-1 Y59C; henceforth, LMN-Y59C), and was shown to compromise striated body muscle integrity in a dominant manner, leading to impaired body movement (Mattout et al. 2011). In both human and worm lamins, the substituted tyrosine is the $\mathrm{d}$ residue of one of the most highly conserved heptad repeats within the $1 \mathrm{~B}$ coiled coil of lamin A/C (Strelkov et al. 2004). The mutant worm protein can still form dimers and filaments, but integrates less stably into the lamin meshwork, and shows more rapid turnover upon FRAP than wild-type LMN-1 (Wiesel et al. 2008). Many of the phenotypes of human EDMD are recapitulated in C. elegans expressing this mutant form in a fusion with GFP (GFP::LMN Y59C) in the background of wild-type lamin (Mattout et al. 2011). In contrast, strains expressing the equivalent fusion with wild-type lamin (GFP::LMN-1; henceforth, LMN-WT) were phenotypically indistinguishable from wild-type (N2) C. elegans (Mattout et al. 2011). In both cases the GFP fusion proteins constitute $<20 \%$ of the total lamin complement throughout embryogenesis and adulthood (Wiesel et al. 2008; Mattout et al. 2011). Coincident with the muscle pathology, the GFP::LMN-Y59C fusion impaired the relocation of an integrated muscle-specific reporter away from the nuclear periphery in muscle cell nuclei (Mattout et al. 2011). This suggests that the mutant lamin hypersequesters or blocks release of this heterochromatic array. However, it was not clear whether this stemmed from a failure to activate the array-borne myo3 promoter or from an enhanced chromatin-LMN interaction. A programmed cell type-specific release of active tissue-specific promoters from the lamina is documented not only for C. elegans (Meister et al. 2010b; Mattout et al. 2011), but also for tissue-specific genes in a range of mammalian cells (Kosak et al. 2002; Ragoczy et al. 2006; Reddy et al. 2008; Meister et al. 2010b).

Here we investigated the role of chromatin organization in EDMD-like muscle phenotypes using this powerful $C$. elegans disease model. We examined how the LMN-Y59C mutation modifies genome organization, gene expression, and muscle morphology. To understand whether the observed disease phenotypes are linked to chromatin misorganization, we coupled the gain-of-function Y59C lamin mutation with down-regulation of other INM proteins, most notably with ablation of the heterochromatin anchor, CEC-4. Indeed, the defects of the LMN-Y59C allele can be compensated by deleting cec-4, or ablating the ability of its chromodomain to tether H3K9memarked chromatin at the INM /Gonzalez-Sandoval et al. 2015). The analysis of lem-2 or emerin null alleles 
suggests that the effects are specific for cec-4. Thus, we identified compensatory cross-talk between the nuclear lamina and a NE-associated CD protein, showing that the latter is able to counteract the EDMD-like defects in muscle integrity associated with a laminopathy mutation. We suggest that the altered association of chromatin with the NE is causal and not simply a consequence of the disease state.

\section{Results}

\section{Mutant lamin alters endogenous genome organization}

In a wild-type $C$. elegans background, the expression of an EDMD-linked mutant lamin (GFP::LMN-1 Y59C or LMNY59C) altered muscle ultrastructure and impaired body muscle function, coincident with the sequestration of a heterochromatic reporter array bearing a muscle-specific promoter, at the INM in muscle cells (see Fig. 1A-C; Mattout et al. 2011). The mislocalized array consists of $\sim 280$ repeats of an insert that encodes GFP-LacI driven from a baf-1 promoter, and RFP under control of the muscle-specific myo-3 promoter (myo-3p) (Fig. 1A; Towbin et al. 2012). In wild-type muscle cells, with or without $L M N$ $W T$ expression, the myo-3p-containing array shifts away from the nuclear periphery upon activation of the muscle-specific promoter (Fig. 1C; Meister et al. 2010b). Low-level expression of LMN-WT has no impact on this array behavior nor does it confer any discernible muscle defect (Mattout et al. 2011). In the LMN-Y59C-expressing strain, on the other hand, the array remains at the nuclear periphery in muscle cells despite myo-3p activation; hence, the term "hypersequestration." We note that the level of GFP-LacI expressed from (and bound to) the reporter is far higher than the GFP signal of the lamin fusion proteins (Fig. 1B).

While the $L M N-Y 59 C$ mutant gain-of-function sequestration of the myo- $3 p$ reporter was intriguing, it was important to determine how LMN-Y59C expression affects the spatial distribution of endogenous chromatin. To test this we performed muscle-specific genome-wide emerin-DamID, a method in which the muscle-specific expression of an INM protein (emerin or EMR-1) fused to the $E$. coli DNA adenine methyltransferase (Dam) enables mapping of chromatin at the INM of muscle nuclei (Gómez-Saldivar et al. 2016; Muñoz-Jiménez et al. 2017). We expressed Dam::EMR-1 in a muscle-specific manner thanks to a FLP-FRT system driven by muscle-specific FLP expression (Fig. 1D; Muñoz-Jiménez et al. 2017). This resulted in the preferred methylation of adenines within GATC motifs in chromatin adjacent to the INM in muscle cells only. The use of $\mathrm{G}^{\mathrm{me}} \mathrm{ATC}$-specific restriction enzymes and ligation-mediated amplification, generated signals that were then normalized to the level of modification upon expression of GFP::Dam alone. The regions enriched for the resulting EMR-DamID signal are equivalent to lamina-associated domains (LADs) mapped by LEM-2 chromatin IP or LMN-1-DamID (González-Aguilera et al. 2014; Gonzalez-Sandoval et al. 2015).
L3/L4 stage larvae expressing $L M N-W T$ or $L M N-Y 59 C$ and muscle-specific Dam::EMR-1 were lysed and the ${ }^{m e}$ A-bearing DNA was digested, sequenced, and normalized to DNA from similarly staged larvae that express nuclear GFP::Dam. We plotted the relative enrichment of perinuclear chromatin ( $\log _{2}$ [Dam::EMR-1/GFP::Dam] referred to as $\log _{2}$ [EMR-1/GFP]) (González-Aguilera et al. 2014). Figure 1E shows normalized enrichments between the LMN-Y59C-expressing strain (blue line) and an isogenic strain expressing the wild-type lamin fusion $L M N-W T$ (black line). Importantly, neither endogenous emerin nor an EMR-1::mCherry fusion showed an altered localization in the $L M N-Y 59 C$ strain (see Supplemental Fig. S1A; Wiesel et al. 2008). Additionally, endogenous levels of $1 m n-1$, $e m r-1$ and $1 e m-2$ are similar in wild-type and the mutant strains used (Supplemental Fig. S1B).

In the LMN-WT muscle cells, the heterochromatin-rich and gene-poor chromosome arms were more closely associated with the INM, while the gene-rich chromosome cores were more central, although the dynamic range of bound and unbound sequences was lower in late larval muscle cells, than in the nuclei of embryos or L1 intestine (cf. Fig. 1E with Gonzalez-Sandoval et al. 2015; Cabianca et al. 2019). This likely reflects active muscle-specific genes found on chromosome arms and technical limitations arising from the nonspherical shape of muscle nuclei. Nonetheless, even the chromosome with the "flattest" pattern, Chr III, showed significantly higher Dam-EMR signal on the chromosome arms than on its core; this trend held up upon averaging across the triplicate DamID assays, for all autosomes (Fig. 1G,H). This is consistent with earlier reports of worm chromosome architecture scored by lamin-DamID and LEM-2 ChIP in whole embryos (Ikegami et al. 2010; Towbin et al. 2012; González-Aguilera et al. 2014; Gonzalez-Sandoval et al. 2015), although the difference between chromosome arms and cores is less pronounced.

In mutant L3/L4 larvae expressing LMN-Y59C we observed subtle but reproducible changes in muscle cell LADs (Fig. 1E,F). When scored by $100-\mathrm{kb}$ bins, $28.5 \%$ showed a shift towards the NE (higher [EMR-1/GFP] values), while $20.7 \%$ shifted inward (Supplemental Fig. S1C). Intriguingly, $90.2 \%$ of the sequences that gained perinuclear signal were found on chromosome arms (distal $\sim 5 \mathrm{Mb}$ ); while $58.4 \%$ of the bins shifting inwards map to gene-rich chromosomal cores (Supplemental Fig. S1C,D). The average change across an autosome (Chr III) split into right, left, and core domains, is shown in Figure $1 \mathrm{G}$. In brief, the LMN-Y59C mutation tended to enhance $\mathrm{NE}$-association of sequences on arms and to reduce it for chromosomal cores.

To quantify the changes in peripheral binding sites, we next examined 10-kb domains or "bins," as they are more likely to reflect the behavior of individual genes and promoters. We grouped all 10-kb bins from both mutant LMN-Y59C and LMN-WT samples that showed a $\log 2$ (EMR-1/GFP) ratio $>3$ for LMN-Y59C-LMN-WT 1645 bins), and asked whether they were already perinuclear in $L M N-W T$-expressing worms. This was not the case (Fig. 1I), arguing that the $L M N-Y 59 C$ mutant induces the 
Downloaded from genesdev.cshlp.org on April 26, 2023 - Published by Cold Spring Harbor Laboratory Press

LAD misorganization and its suppression in EDMD
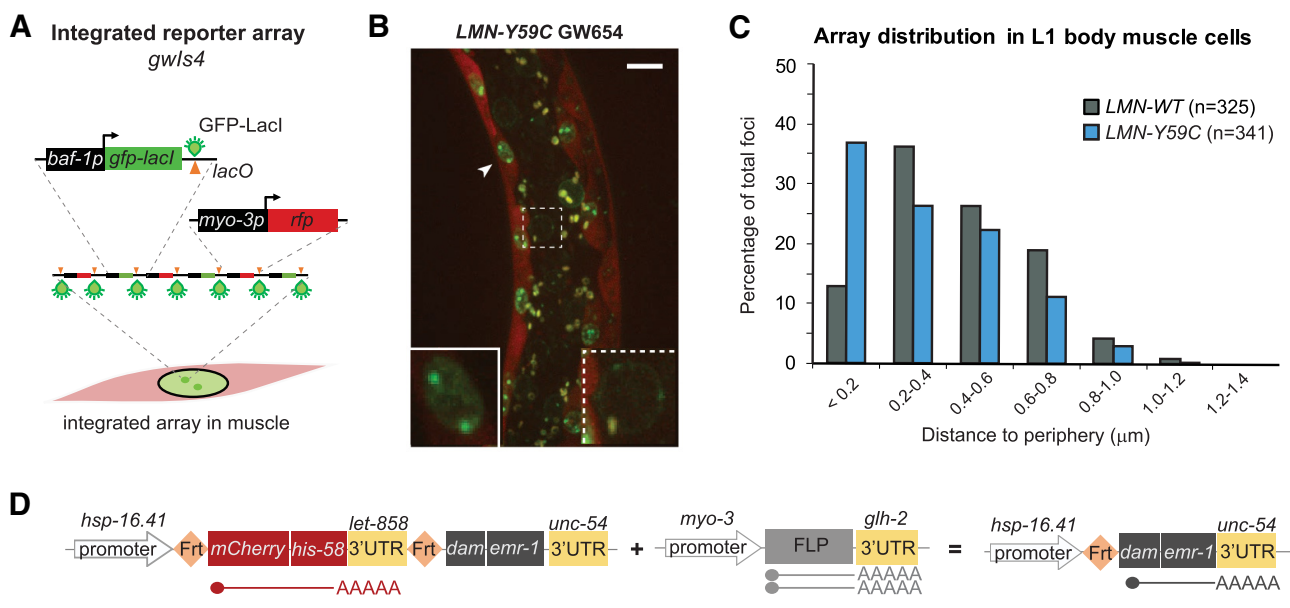

E
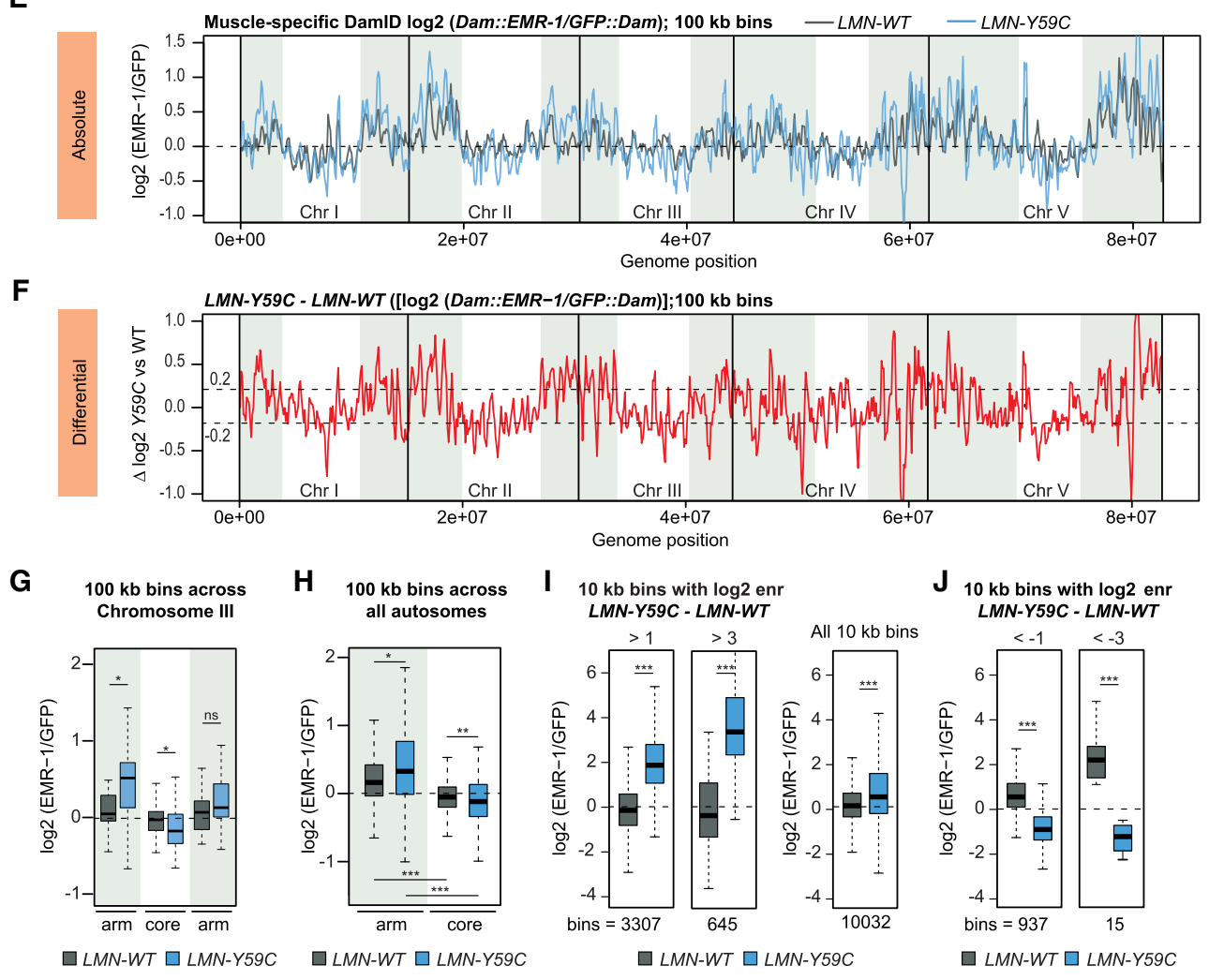

Figure 1. Mutant lamin (LMN-Y59C) leads to alterations in genome organization. (A) Schematic of the array gwIs4 (Meister et al. 2010b). (B) Representative image of C. elegans L1 larvae expressing LMN-Y59C (GW654) with an integrated gwIs4 array. (Arrowhead) Muscle nucleus in inset; (dotted box) intestine nucleus with the gwIs 4 array at NE. Low GFP: :LMN-Y59C expression marks the NE. Scale bar, $5 \mu$ m. (C) Quantitation of the gwIs4 focus distance from NE in muscle cells of $L M N-W T$ (GW 656) versus Y59C-LMN (GW654). Shortest path from the gwIs 4 focus center to NE is scored $(n=$ foci scored). $L M N-W T$ versus $L M N-Y 59 C P<0.0001$, Chi-squared test (peripheral $<0.2 \mu \mathrm{m}$ vs. central $>0.2 \mu \mathrm{m})$. $(D)$ Single-copy transgene insertions for muscle-specific emerin DamID. (Left) Basal hsp-16.41p activity produces very low-level mCherry::his-58 transcript from an FRT-flanked stop cassette. (Right) In muscle cells, FLP recombinase expressed from myo-3p leads to stop cassette excision and low-level Dam::EMR-1 expression. (E) Line plot of $\log _{2}$ Dam::EMR-1 normalized to GFP::Dam in $L M N-W T$ (black) and LMN-Y59C (blue) in L3/L4 C. elegans muscle. Signals are averaged over three sliding 100-kb windows. Chromosome arms (see the Materials and Methods) are shaded. The dotted line indicates zero $\log _{2}$ ratio. (F) Line plot of $L M N-Y 59 C-L M N-W T$ $\left(\log _{2}\right)$ showing differential EMR-Dam modification in $L M N-Y 59 C$ versus $L M N$-WT. Dotted line indicates 0.2 and -0.2 thresholds. (G) Box plots comparing the frequency of contacts of Chr III ( $\log _{2}$ [EMR-1/GFP]) in $L M N-W T$ versus $L M N$-Y59C, averaged over three sliding 100-kb windows. $\left.\left(^{*}\right) P<0.01 ;\left(^{* *}\right) P<0.0001 ;{ }^{* * *}\right) P<10^{-10}$; (ns) nonsignificant using Wilcoxon rank sum tests. $(H)$ As in $G$, but EMR-Dam contact frequency across all autosomal arms and cores is shown ( $\log _{2}$ [EMR-1/GFP]) in $L M N-W T$ and $L M N$-Y59C, averaged over three sliding 100-kb windows. (I) Box plots comparing the frequency of contacts ( $\left.\log _{2}[\mathrm{EMR}-1 / \mathrm{GFP}]\right)$ for $10-\mathrm{kb}$ bins that are enriched (enr) over a threshold of either $>1$ or $>3$ for the difference $\log _{2}(\mathrm{LMN}-\mathrm{Y} 59 \mathrm{C})-\log _{2}(\mathrm{LMN}-\mathrm{WT})$. (J) As $I$ but comparing the bins for which the values $\log _{2}$ (EMR-1/GFP) are below the threshold of -1 or -3 for the difference $\log _{2}(\mathrm{LMN}-\mathrm{Y} 59 \mathrm{C})-\log _{2}(\mathrm{LMN}-\mathrm{WT})$. 
formation of LADs de novo, rather than simply reinforcing existing contacts. A similar conclusion was drawn for a larger selection of fragments that shift to the periphery with $\log _{2}$ ratios $>1$ for $L M N-Y 59 C-L M N-W T(n=3307)$. Intriguingly, although the sequences that shifted towards the periphery in the mutant were slightly more enriched in repetitive DNA elements (consistent with their localization along chromosomal arms; Liu et al. 2011), they did not show a striking enrichment for the histone modification H3K9me2 or H3K9me3 based on whole L3 larval ChIP-seq (Supplemental Fig. S1E,F). Finally, we identified sequences that shifted away from the NE in the LMNY59C mutant $\left(\log _{2}[\right.$ EMR-1/GFP $]<-1$ or -3$)$. For this less-abundant subset, primarily found in chromosomal cores, the LMN-Y59C mutation appears to have the opposite effect; i.e., it counteracted peripheral anchoring (Fig. 1J). Still, none showed significant enrichment for repeats or H3K9me2/3 (Supplemental Fig. S1E,F).

To understand what the newly acquired LADs $\left(\log _{2}\right.$ [EMR-1/GFP] >1 for LMN-Y59C-LMN-WT) had in common, we screened them for abundant transcription factor motifs. Two of the most highly enriched consensus motifs are recognized by the transcription factor EFL-2, one of two worm E2F homologs, EFL-1 and EFL-2, both of which are bound and inhibited by the retinoblastoma-like pocket protein $\mathrm{Rb}$ (LIN-35 in C. elegans) (Supplemental Fig. S2). Intriguingly, the set of $10-\mathrm{kb}$ bins that shifted inward were also enriched for EFL-2 binding sites (Supplemental Fig. S2). Most of EFL-1 is found in complex with DP-1 and the MuvB complex, which recognizes a larger bipartite motif that was not enriched in the LMN-Y59C-sensitive LADs (Supplemental Fig. S2; Narasimhan et al. 2015). It is not known whether EFL-2 is found in multiple complexes, but the fact that its consensus is enriched on sequences that shift position suggests that at least a subset of EFL-2-Rb complexes may be affected by the LMNY59C mutation. The presence of binding motifs for an $\mathrm{Rb}$-binding E2F homolog is likely to be relevant, given earlier work showing that mammalian lamin A binds the hypophosphorylated form of Rb (Pekovic et al. 2007). Moreover, the repression of E2F by hypophosphorylated $\mathrm{Rb}$ is thought to be needed to arrest myoblast proliferation sufficiently to allow differentiation (Frock et al. 2006; Melcon et al. 2006; Pekovic et al. 2007; Elenbaas et al. 2018). In mammals, the interaction of $\mathrm{Rb}$ with lamin $\mathrm{A}$ and LAP2 $\alpha$ was altered by certain lamin A/C mutants and by the loss of emerin or LAP2a (Markiewicz et al. 2002; Melcon et al. 2006; Pekovic et al. 2007).

\section{Loss of CEC-4, but not LEM-2 or emerin, derepresses a heterochromatic reporter}

The nuclear lamina is a collection of lamin- and INMinteracting proteins that form a meshwork of proteins underlying the nucleoplasmic side of the inner nuclear membrane. Some lamin components, such as LAP2a and lamin A in mammals, are also found in the nuclear interior, to varying degrees. C. elegans has most of the major nuclear lamina components found in mammals-i.e., lamin, emerin (EMR-1), SUN domain proteins, and the
LEMD2/MAN1 homolog LEM-2 (Fig. 2A) —but it lacks two mammalian components that were previously implicated in muscle differentiation (LAP2 $\alpha$ and the lamin B receptor or LBR). On the other hand, worms have a highly specific, NE-associated H3K9 methylation anchor called CEC-4 (Gonzalez-Sandoval et al. 2015).

Given that mutations in either emerin or lamin A can generate EDMD in humans, we asked whether the loss of other INM components of C. elegans mimic the nuclear changes observed with the LMN-Y59C allele in muscle cell nuclei. Using a standardized, high-throughput image processing pipeline that segments and analyzes confocal 3D fluorescence imaging data in an automated fashion (Fig. 2B), we examined the effects of EMR-1, LEM-2, or CEC-4 ablation, compared with the low-level expression of LMN-WT or LMN-Y59C, by monitoring muscle cell nuclei in the appropriate L1 larvae. We scored nuclear size, shape, and the expression of GFP-LacI from the gwIs4 reporter (Fig. 2C-G). Under none of the conditions tested [the presence of mutant lamin (LMN-Y59C) or null alleles cec-4 (ok3214), lem-2 (tm1592), or emr-1 $(g k 119)]$ was nuclear volume significantly altered (Fig. 2G). On the other hand, cec-4 (ok3124) (hereafter cec-4 4 ) led to an increase in the intensity of GFP-LacI at the array focus and throughout the nucleus (Fig. 2D,E), indicative of array derepression. There was no significant change in GFP fluorescence seen in lem-2 (tm1592) worms, while the ablation of emerin [emr-1 (gk119)] had a repressive effect, even stronger than $L M N-Y 59 C$. We could exclude that the phenotypes arise from altered expression of the genes encoding these INM proteins (Supplemental Fig. S1B). In addition to GFP up-regulation, cec- $4 \Delta$ altered the typical oblong shape of muscle nuclei, making them more spherical, while lem-2 (tm1592), emr-1 (gk119), and the expression of the mutant LMNY59C protein did not (Fig. 2F). LMN-WT expression in all cases resembled wild-type (N2) worms. In summary, whereas emerin ablation yielded muscle nuclear defects reminiscent of $L M N-Y 59 C$, cec-4 $\Delta$ had the opposite effect.

CEC-4's role in heterochromatin localization was first identified and characterized in C. elegans embryos, although the protein is present throughout development (Gonzalez-Sandoval et al. 2015). Indeed, in L1 larvae CEC-4 is more abundant in muscle cells than in other tissues (Fig. 3A; Gonzalez-Sandoval et al. 2015). Given its abundance and impact on muscle nuclear morphology (Fig. 2F), we examined whether CEC-4 plays a role in sequestering heterochromatic arrays at the nuclear periphery in muscle. To do this, we monitored the position of the heterochromatic array caIs3, which contains the promoter pha-4p, which is silent in both muscle and hypoderm (Fig. 3B). We scored the position of the GFP-LacItagged cals 3 reporter in the nuclei of differentiated tissues of both wild-type and $c e c-4 \Delta$ larvae, relative to emerinmCherry, a marker for the INM. In muscle cells, the cec- $4 \Delta$ mutation compromised the peripheral enrichment of the array $(80 \%$ of 813 foci in wild type, vs. $57 \%$ of 1037 in cec-4 $)$ (Fig. 3C,D), demonstrating a role for CEC-4 in heterochromatin tethering beyond embryogenesis. This is consistent with effects monitored for cec-4 $\Delta$ in the 

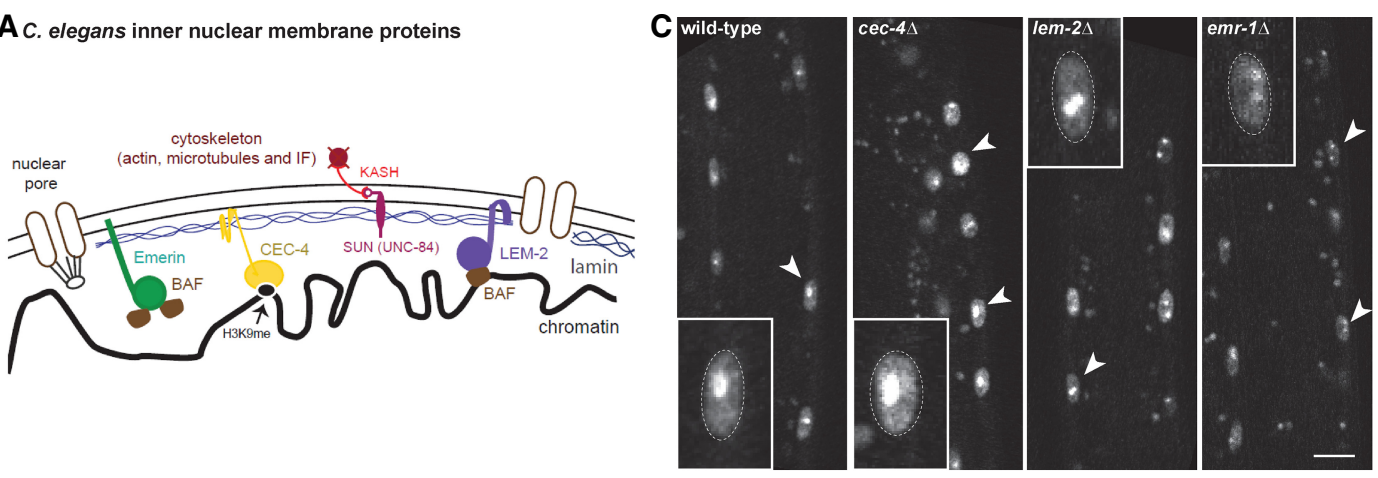

B High-throughput image analysis
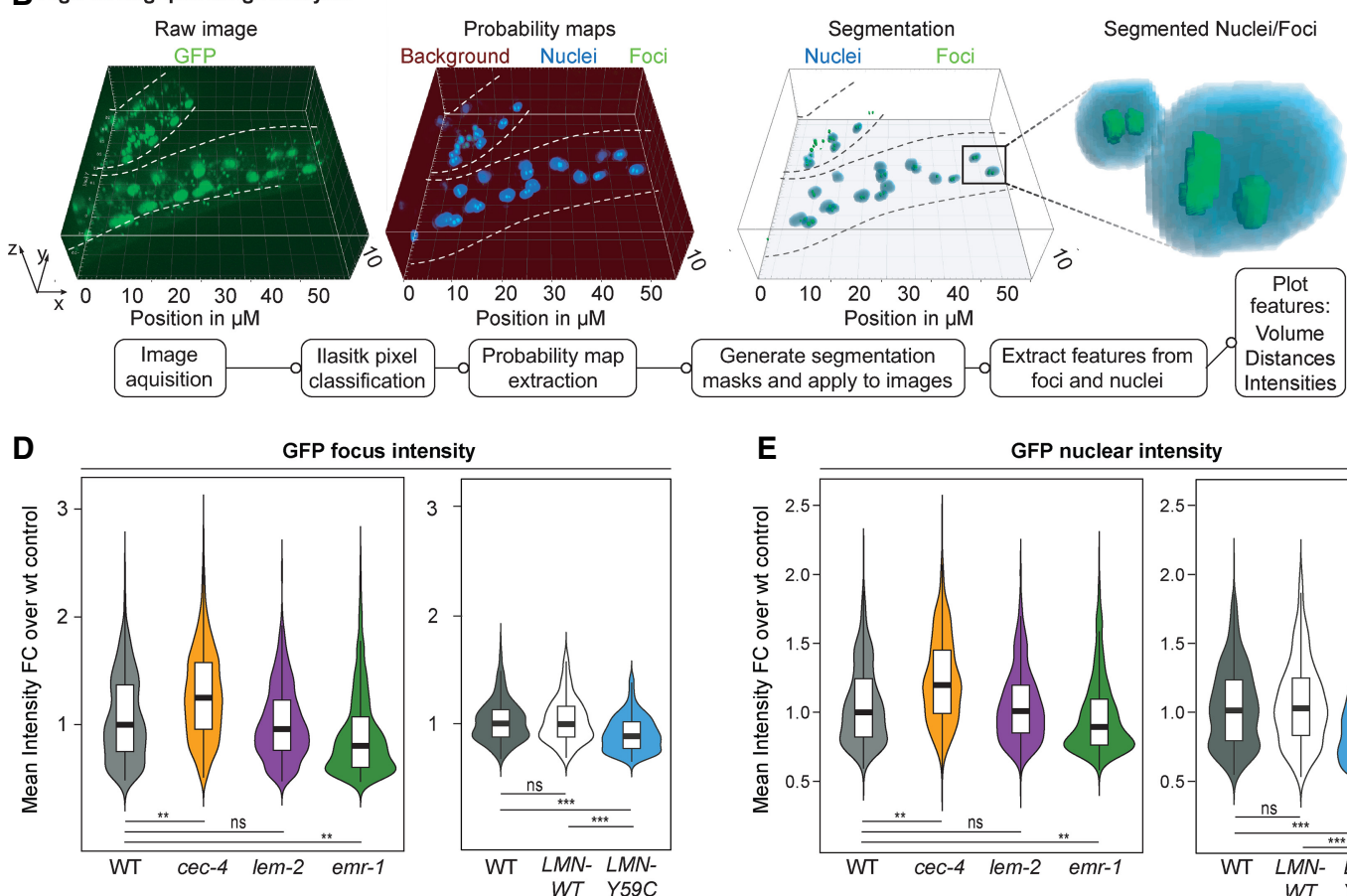

E


$\square$ wild-type $(\mathrm{WT} ; \mathrm{n}=363 / 617) \square c e c-4$ (ok3214) (n=695) $\square$ lem-2 (tm1582) (n=664) $\square e m r-1$ (gk119) (n=582) $\square L M N-W T(\mathrm{n}=776) \quad \square L M N-Y 59 C(\mathrm{n}=565)$

Figure 2. INM proteins EMR-1, LEM-2, and CEC-4 have distinct effects on array expression and nuclear shape. (A) Sketch of the C. elegans INM components (Bank and Gruenbaum 2011a; Cohen-Fix and Askjaer 2017). (B) Summary of automated image segmentation and analysis used in D-G. (C) Examples of confocal fluorescence imaging of C. elegans L1 larvae [wild type; gwIs4 (GW76), cec-4L; gwIs4

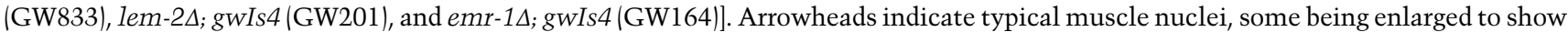
intensity and position of the gwIs 4 array. Scale bar, $5 \mu \mathrm{m} .(D-G)$ Violin plots resulting from the automated analysis monitoring GFP-LacI foci intensity $(D)$, total nuclear GFP-LacI intensity $(E)$, muscle nuclear shape $(F)$, or volume $(G)$. Strains used are indicated below the box plots; the mutants are null alleles, except for strains expressing $L M N-W T$ (GW656) or LMN-Y59C (GW654) from integrated arrays. These

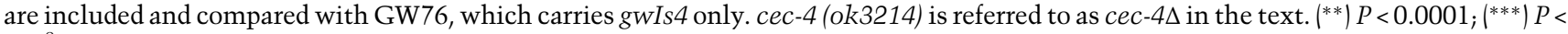
$10^{-8} ;$ (ns) nonsignificant, versus wild type, Tukey post-hoc tests. 
A

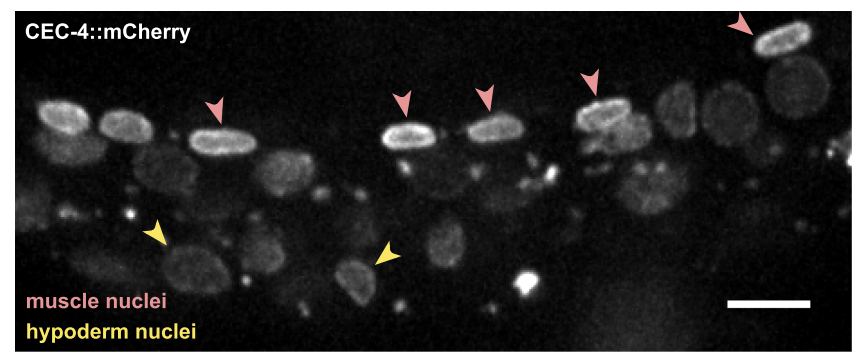

C
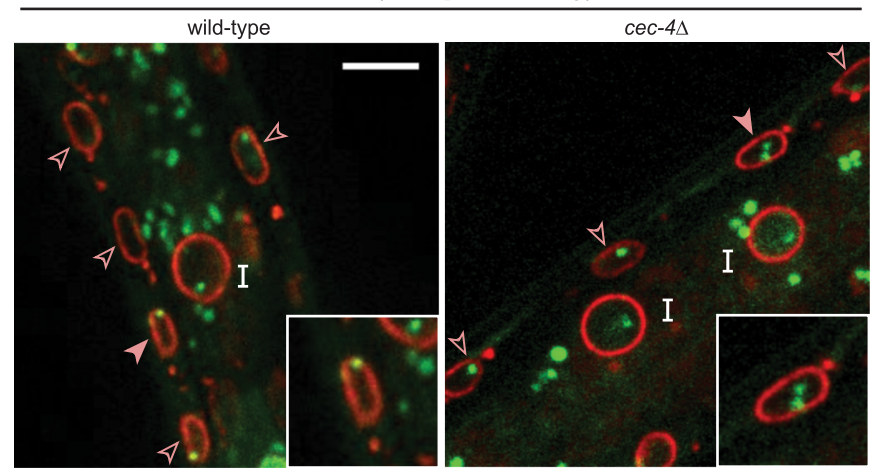

E

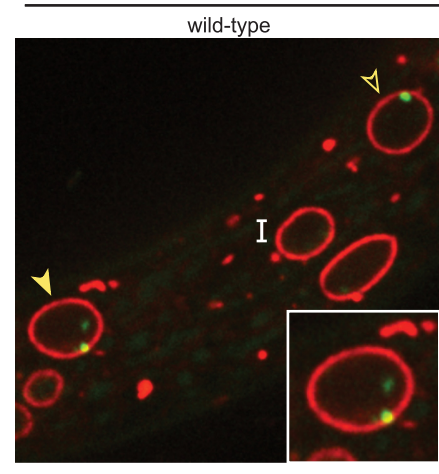

B Heterochromatic reporter array: cals3 [pha-4p] O. GFP::Lacl $\triangle$ lacO

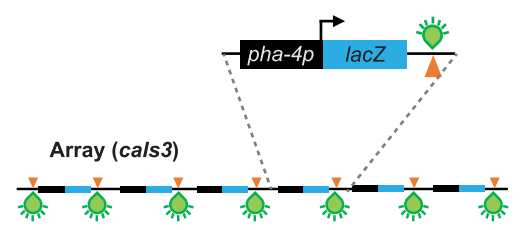

D

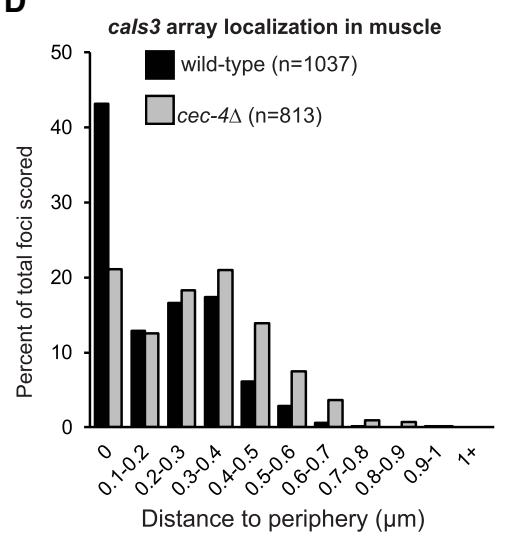

$\mathbf{F}$

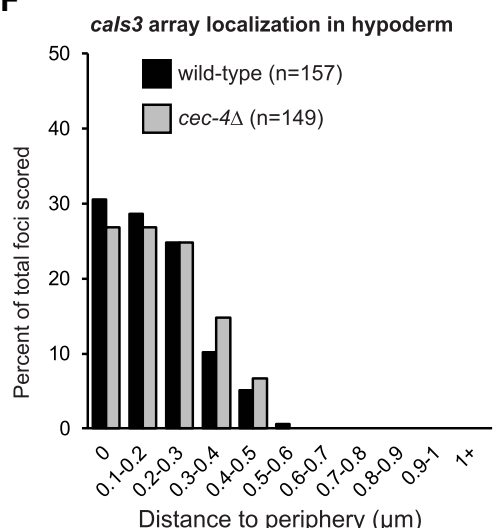

Figure 3. CEC-4 contributes to heterochromatin perinuclear sequestration in muscle. (A) Fluorescence microscopy of a C. elegans L1 larvae expressing CEC-4: :mCherry capturing both muscle (pink arrow) and hypoderm (yellow arrow) nuclei. Average mCherry signal intensity $=2.1$ arbitrary units (a.u.) for muscle, 1.5 a.u. for intestine, and 1.9 a.u. for hypoderm (Gonzalez-Sandoval et al. 2015). The larvae is twisted such that muscle nuclei appear closer together than usual. Scale bar, $5 \mu \mathrm{m}$. (B) Sketch of the caIs3 reporter integrated in 200 copies. This array expresses LacZ from the gut-specific pha-4 promoter and contains one lacO consensus per repeat. A second integrated array (gwIs39) expresses GFP::LacI from baf-1p and lacks lacO. $(C, E)$ Fluorescence confocal images of $C$. elegans L1 larvae expressing the pha-4 reporter (cals3; green) in either wild-type (GW1394) or cec-4 (ok3124; cec-44; GW1395) worms expressing EMR-1::mCherry (red). Arrowheads indicate muscle cells $(C)$ and hypoderm cells $(E)$ and " $\mathrm{I}$ " indicates intestine cell nuclei. Filled arrowheads mark nuclei in insets. Scale bar, $5 \mu \mathrm{m} .(D)$ Quantitation of subnuclear positioning of the cals3 array in muscle cells of wild-type or cec-4 $\Delta$ larvae from at least two biological replicates. Wild type versus cec- $4 \Delta P<0.0001, \chi^{2}$ (peripheral $<0.2 \mu \mathrm{m}$ vs. central $\left.>0.2 \mu \mathrm{m}\right)$. $(F)$ As in $D$ but for hypoderm cells. $P$-value wild type versus $c e c-4 \Delta$ is not significant.

peripheral anchoring of endogenous sequences in L1 intestinal cells, although silent arrays in these cells remained peripheral (Cabianca et al. 2019). The silent caIs3 array in hypodermal nuclei behaved similarly: The arrays remained at the NE of cec-4A larvae (Fig. 3E,F; Supplemental Fig. S3A,B). In conclusion, CEC-4 has exceptionally high levels in differentiated muscle cell nuclei and is ac- tive in heterochromatin retention at the NE in muscle, where it also contributes to array repression and the maintenance of nuclear shape.

Given that the effects of $c e c-4 \Delta$ on the reporter in muscle were often the opposite of those scored in $L M N-Y 59 C$ expressing worms, we examined the effect of combining the two mutations in one strain (Fig. 4). Remarkably, in 
A

A gwls4 foci visualized in L1 muscle nuclei

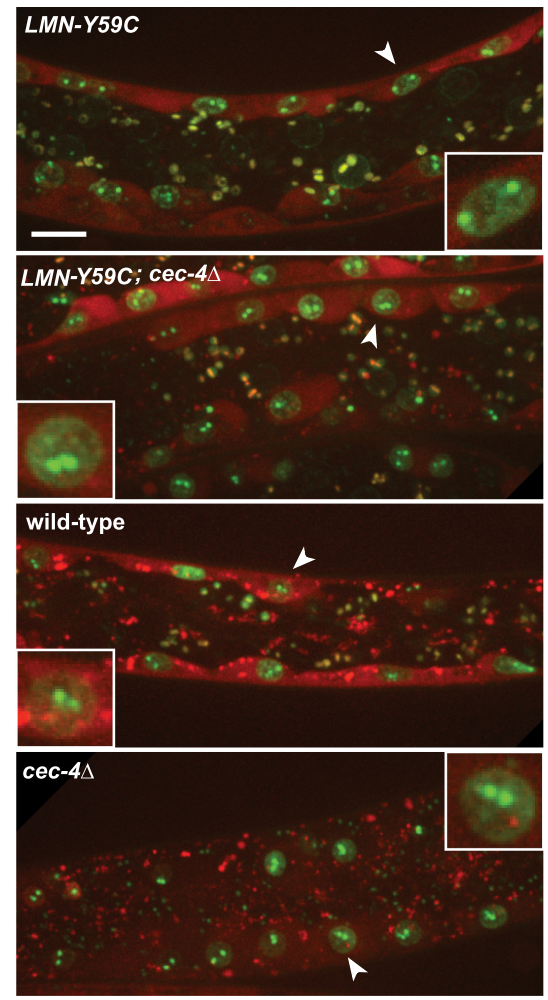

B gwls4 distance from the NE in L1 muscle nuclei
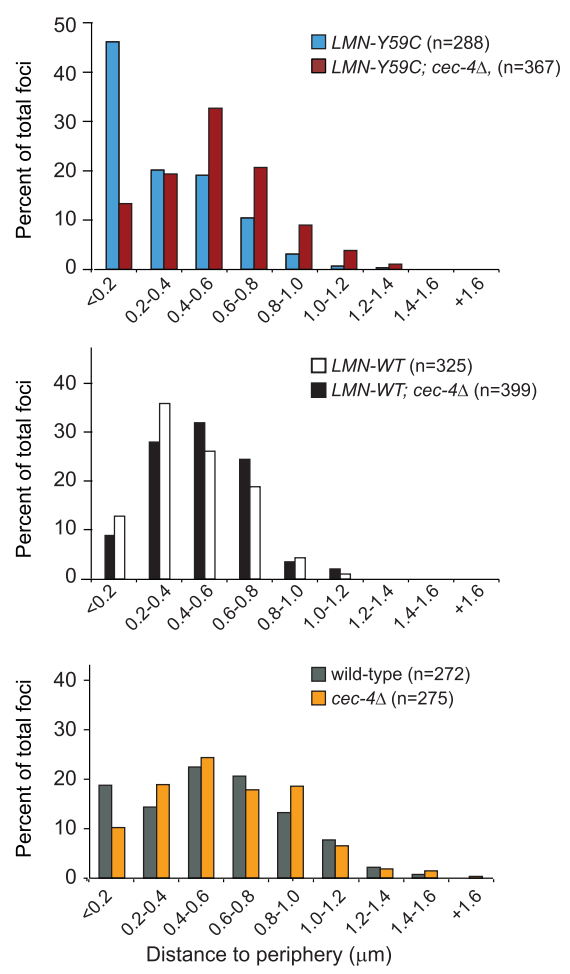

C
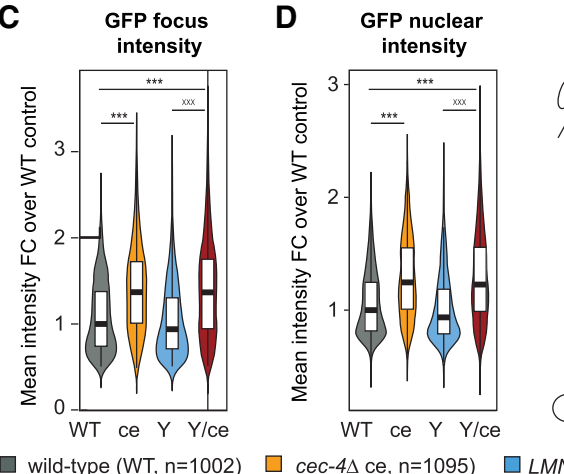

$\square$ cec- $4 \Delta$ ce, $n=1095)$

E Nuclear shape

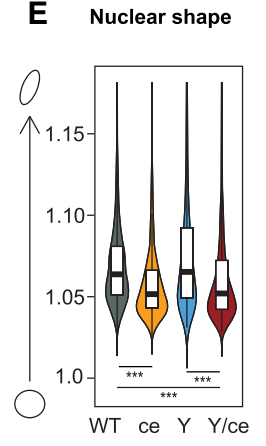

F Nuclear volume

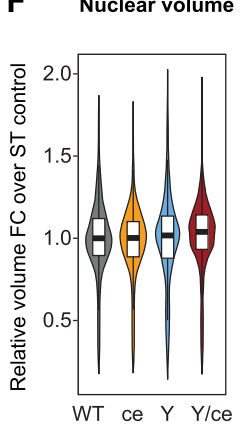

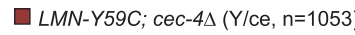

Figure 4. Altered array positioning in the $L M N-Y 59 C$ mutant is reversed by cec-4 $\Delta$. (A) Confocal fluorescence microscopy of $C$. elegans muscle nuclei bearing the muscle-specific gwIs 4 array and expressing $L M N-Y 59 \mathrm{C}$ in wild-type or cec-4 $\Delta$ backgrounds, as indicated $[L M N$ -

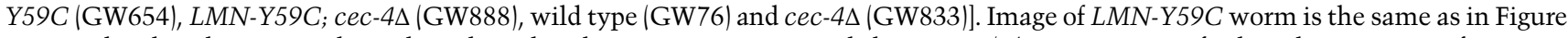
1. Arrowheads indicate muscle nuclei enlarged to show array position. Scale bar, $5 \mu \mathrm{m}$. $(B)$ Quantitation of subnuclear position of $g w I s 4$ in muscle cells of indicated genotypes from at least two biological replicates. Distances measured are shortest distance from focus to the NE. (n) Number of nuclei scored. $L M N$-Y59C versus $L M N-Y 59 C$; cec- $4 \Delta, P<0.0001 ; L M N-W T$ versus $L M N-W T$; cec-4 $\Delta, P>0.01$; WT versus cec- $4 \Delta, P=0.004$, by $\chi^{2}$ test (peripheral $<0.2 \mu \mathrm{m}$ vs. central $\left.>0.2 \mu \mathrm{m}\right)$. $(C-F)$ Violin plots for $g w I s 4$ focus GFP-LacI intensity. (WT) Wild

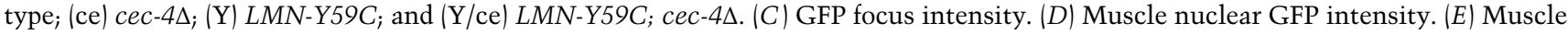
nuclear shape/roundness. $(F)$ Muscle nuclear volume. $(n)$ Number of nuclei counted for each strain. $P$-values were calculated and are indicated as in Figure 2, D-G

the double mutant, the LMN-Y59C-mediated gwIs4 array sequestration at the NE was reversed: The deletion of cec-4 restored an internal localization for the active myo-3p-containing array, as was seen in $\mathrm{N} 2$ and/or $L M N-W T$ muscle nuclei (Fig. 4A,B). In $L M N-W T$ or $\mathrm{N} 2$ backgrounds, since in both cases the gwIs 4 array was already internal, cec- $4 \Delta$ had very minor effects on array lo-

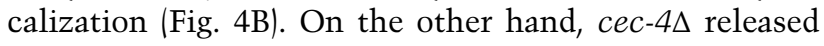

the heterochromatic from the NE in early embryos expressing $L M N-Y 59 C$, as it did in wild-type embryonic nuclei (Supplemental Fig. S3C,D). We subjected the double mutant to the high-throughput analysis of GFP intensity, nuclear volume, and nuclear shape, as carried out in Figure 2, and found that the coupling of cec-4 $\Delta$ with $L M N$ Y59C enhanced the GFP-LacI signal on the array and throughout the nucleus (Fig. 4C,D). Moreover, muscle 
nuclear shape became more spherical, although nuclear volume was unchanged (Fig. 4E,F). Again, we could rule out indirect changes due to lamin misregulation in the single or double mutant (Supplemental Fig. S1A,B). In summary, cec- $4 \Delta$ suppressed or counteracted the hypersequestration of a muscle-specific promoter-carrying array (myo-3p on gwIs4) in muscle nuclei, provoked by the gain-of-function LMN-Y59C mutation.

Loss of cec-4 specifically reverses the Y59C mutant defects in mobility and muscle structure

Given its suppression of the $L M N-Y 59 C$-induced array phenotype, we next asked whether cec-4 $\Delta$ suppresses defective muscle function as well. To this end, we examined head thrashing and muscle structure in C. elegans, in both single- and double-mutant backgrounds. Previous work showed that the ectopic expression of LMN-Y59C altered actin filament organization and compromised mobility, which is monitored by the frequency of rapid head movements to the right when adult worms are placed in liquid (Fig. 5A; Mattout et al. 2011). We tested L4 larvae from wild-type, cec-4 $, L M N-W T, L M N-Y 59 C$, and $L M N-Y 59 C$; cec- $4 \Delta$ double-mutant strains, scoring head bend frequency during $30 \mathrm{sec}$. Populations from at least six independent assays were quantified, and to minimize variability, only three genotypes were measured in parallel. In addition, strains with and without the gwIs 4 array were monitored. We found no significant difference between wild-type, $L M N-W T$, and cec-4 4 worms (mean head bend frequency ranged from 53.7 to 58.9 in different assays, $[P>0.5])$; however, we scored a significant reduction of head bend frequency in the mutant LMN-Y59C (34.6; $P<0.01$ as compared with wild type) (Fig. 5B). Importantly, combining cec-4D with $L M N-Y 59 C$ restored rates of head-thrashing equivalent to either wild type or the cec- $4 \Delta$ single mutant (Fig. 5B).

Restoration of muscle function by cec-4 $\Delta$ correlates with restored sarcomere structure

To link the defects in body muscle function in the single and double mutants (LMN-Y59C vs. LMN-Y59C; cec-4D) to muscle anatomy, we used fluorescence imaging to monitor both actin and myosin organization in the sarcomeres of young adult C. elegans. Sarcomeres consist of a bundle of thick filaments (myosin) and thin filaments (actin) that are interdigitated. Using young adults, we could easily score changes in actin organization of muscle cells by tracking high-resolution images of phalloidin-rhodamine-stained striated muscle (Fig. 5C-E). We scored actin organization in each sarcomere as normal or abnormal, with the latter presenting disordered filaments (waves, gaps, and fusions in the actin filaments, Fig. 5C). To convince ourselves that these reflect sarcomere pathologies and not artefacts, we compared phalloidin staining of wild-type and $L M N-W T$ or $L M N-Y 59 C$-expressing worms with the same structures stained in $\mathrm{N} 2$ worms treated with RNAi against unc-15 or unc-45 (Waterston et al. 1977; Venolia and Waterston 1990). These genes are in- volved in the enervation of muscle and lead to worms that cannot move (Waterston et al. 1977; Venolia and Waterston 1990). Whereas the phalloidin staining of wild type and $L M N-W T$ (with and without control RNAi) revealed uniform and smooth actin filaments, the treatment with unc-15 or unc-45 RNAi showed extensive waves, ripples, breaks, and irregularities in the actinmyosin filaments (Fig. 5C). The LMN-Y59C worms (with and without control RNAi) showed a much less pronounced, but reproducible, presence of similar fractures, waves, and irregularities within sarcomeres and at sarcomere junctions (Fig. 5C,D). The abnormalities were subtle but consistently observed in $L M N-Y 59 C$, unlike $L M N-W T$ and N2 worms. We quantified the frequency of aberrant sarcomeres, comparing wild-type, cec-4D, LMN-Y59C, and the $L M N-Y 59 C$; cec- $4 \Delta$ double-mutant strains. The percentage of sarcomeres containing abnormal actin organization increased robustly only in the $L M N-Y 59 C$ strain $(P<0.001$; Fig. $5 \mathrm{E})$, and by combining cec- $4 \Delta$ with $L M N-Y 59 C$ we found the higher frequency of waves, ripples, and breaks (Fig. 5E), as well as the defects scored at sarcomere junctions (Supplemental Fig. S4A,B, arrowheads), to be suppressed. To ensure that this phenomenon was not influenced by the fixation required for phalloidin staining, we monitored sarcomere structure by a GFPtagged muscle-specific myosin expressed in the same mutants. Live imaging of young adult $C$. elegans expressing GFP::MYO-3 showed abnormal myosin organization, particularly at sarcomere junctions, and uniquely in muscle of the LMN-Y59C worms (Supplemental Fig. S4C,D). Once again, the defect was suppressed by the deletion of $c e c-4$.

As shown above, the LMN-Y59C and cec-4 mutant effects are most pronounced in muscle and may be cell type-specific (cf. Fig. 3). To see whether the removal of another INM protein would confer or suppress these EDMDlike phenotypes, we examined the deletion of $1 \mathrm{em}-2$ (human LEMD2 or MAN-1) in more detail, scoring for both functional and structural defects in muscle. Unlike cec-4, we found that 1em-2 deficiency on its own reduced head-thrashing rates, very much like the Y59C-LMN mutant (mean head bends to the right: $1 e m-2 \Delta=37.5$ vs. $L M N-Y 59 C=34.7$ ) (Supplemental Fig. S4E; see also Barkan et al. 2012). Interestingly, the combination of $1 e m-2$ with $L M N-Y 59 C$ showed epistasis, rather than suppression of the muscle defects (mean head bends 40.9, $P>$ 0.5) (Supplemental Fig. S4E). Moreover, a detailed analysis of sarcomere and junction integrity by phalloidin staining showed no significant variance from wild type for either phenotype in lem-2 deficient worms (Supplemental Fig. $\mathrm{S} 4 \mathrm{~F})$. We conclude that the $L M N-Y 59 \mathrm{C}$-induced defect in sarcomere structure that can be reversed by cec- $4 \Delta$ is not mimicked by $1 e m-2 \Delta$.

To see whether the cec-4 $\Delta$ suppression stems from the loss of binding to the H3K9 methylation mark, we made use of a strain expressing a mutant form of CEC-4 in which the two crucial tyrosine residues of its chromodomain (Y87/Y111) have been replaced by alanines. These substitutions impair binding to the methylated histone H3K9 peptide in vitro, and are sufficient to release 
A

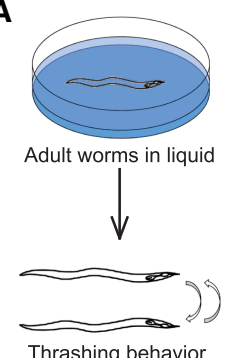

C Phalloidin labeling of actin LMN-WT; RNAi: control

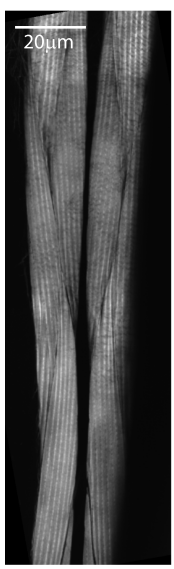

D

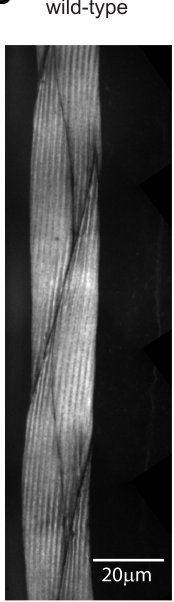

$L M N-W T$
B

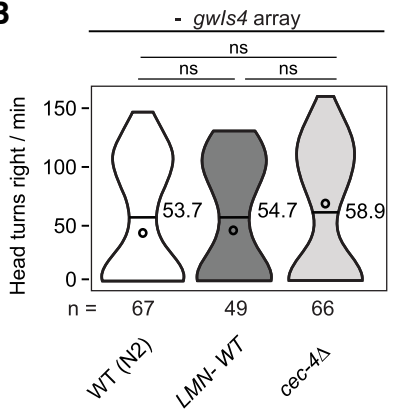

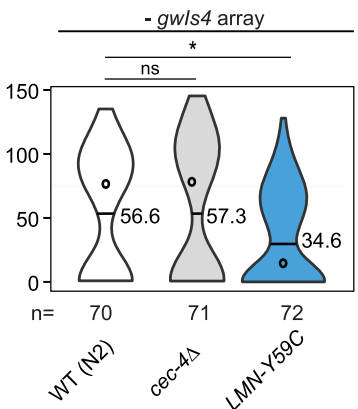
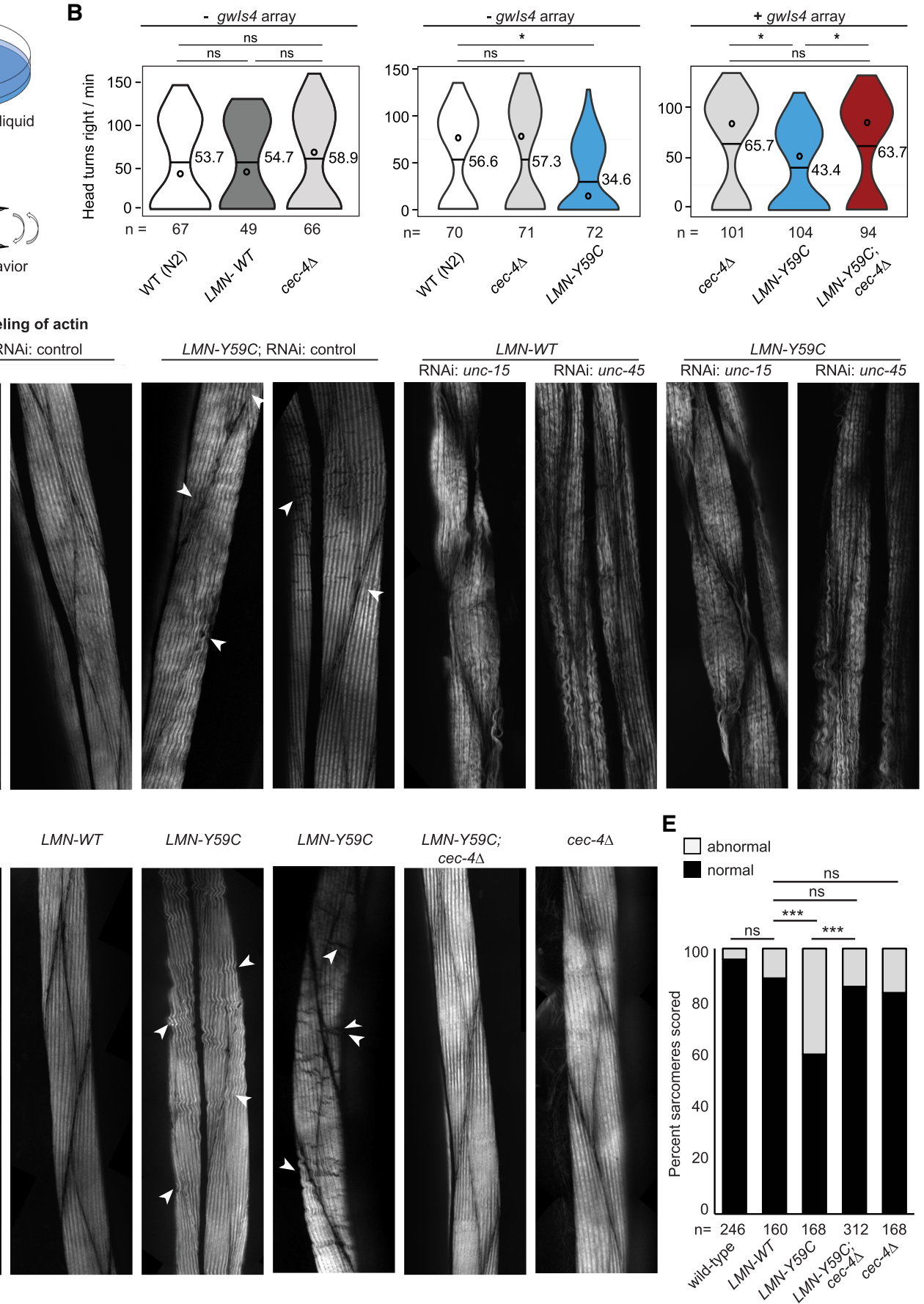

Figure 5. Motility and actin filament organization disrupted by $L M N-Y 59 C$ can be rescued by cec-4 4 . (A) Sketch of the head thrashing (swimming) assay using L4 larval stage worms (see Materials and Methods). (B) Left, individual C. elegans L4 larva of wild-type (N2), LMN-

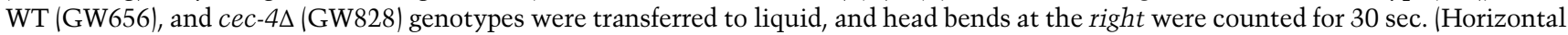
bar) Mean; (open circle) median. Data are from at least three independent assays. Grouped genotypes were quantified in parallel. Middle graph as left, with strains wild type (N2), cec-4 (GW828), and $L M N-Y 59 C$ (GW653). None of these strains carry gwIS4. (Right) Swimming

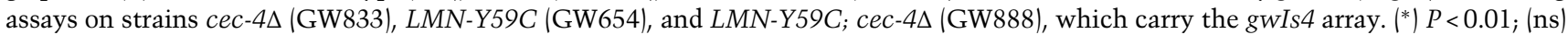
nonsignificant by Student's $t$-test. (C) Representative images of phalloidin-rhodamine staining in L4/young adult C. elegans, illustrating normal and abnormal phenotypes. RNAi for control, unc-15, or unc-45 was done in either a LMN-WT (GW656) or LMN-Y59C (GW653) background. Arrowheads indicate mild abnormal phenotypes in mutant lamin strains. Scale bar, $20 \mu \mathrm{m}$. $(D)$ Representative images of phalloidin-rhodamine staining in L4/young adult C. elegans from wild type (N2), cec-44 (GW828), LMN-WT (GW655), LMN-Y59C (GW653), and $L M N-Y 59 C$; cec-4A (GW1468). Arrowheads and bar are as above. $(E)$ Quantitation of actin organization in muscle cells of indicated strains from three or more biological replicas. $\left.{ }^{* * *}\right) P<0.0001$; (ns) nonsignificant $(P>0.01)$, by $\chi^{2}$ tests. 
heterochromatin from the INM in embryos (Supplemental Fig. S5A; Gonzalez-Sandoval et al. 2015). The LMN-Y59C mutant in a cec-4-null background was complemented either with the Y87A/Y111A mutant form (cec-4(cd2YA):: mCherry) or with wild-type CEC-4 (cec-4::mCherry). The expression of the CD-deficient cec-4(cd2YA) resulted in a partial suppression of the $L M N-Y 59 C$ phenotypes in the head-thrashing assay (Supplemental Fig. S5B), while the WT control (cec-4::mCherry) did not (Supplemental Fig. S5B). Suppression was also scored for the $L M N$ Y59C-induced sequestration of the gwIs4 array in muscle nuclei (Supplemental Fig. S5C,D, green bars). We conclude that loss of the CEC-4-H3K9me binding helps suppress the $L M N-Y 59 C$ phenotypes, although another presently unknown function of CEC-4 may also contribute.

\section{Altered genome organization in the LMN-Y59C mutant is rescued by loss of CEC-4}

Given the ability of cec- $4 \Delta$ to suppress the muscle defects of the $L M N-Y 59 C$ mutant, we asked whether cec-4 $\Delta$ acts by rescuing or suppressing the misorganization of genomic sequences that we scored by muscle-specific EMRDamID in the LMN-Y59C mutant (Fig. 1). We performed the same EMR-DamID in worms expressing either $L M N-Y 59 C$ or $L M N-W T$ coupled with cec-4D. We confirmed that neither the expression nor the localization of emerin or lamin itself was altered by cec-4 $\Delta$ (Fig. 3C, E; Supplemental Fig. S1A,B). Still, the combination of $L M N-Y 59 C$ and cec-4D elevated the EMR-Dam signals in chromosomal cores, and did not only reduce LAD signals, as one might have expected from previous work in embryos (Fig. 6A,B; Gonzalez-Sandoval et al. 2015). Indeed, as many $100-\mathrm{kb}$ bins shifted outwards as inwards upon cec-4 ablation in the LMN-Y59C background (Supplemental Fig. S6A). Using a low-level cutoff $\left(\log _{2}\right.$ ratio < -0.2 ), $92.6 \%$ of the bins shifting inward in cec- $4 \Delta$ were found on chromosome arms, while most 100-kb bins shifting toward the periphery map to chromosomal cores (Supplemental Fig. S6B-D). A similar trend was observed for cec-4 $\Delta$ in the $L M N-W T$ background (Supplemental Fig. S6B-D). At higher resolution (i.e., 10-kb bins), the cec- $4 \Delta$ effect was even more pronounced. We focused on the $10-\mathrm{kb}$ bins that were identified as having a strong shift either toward or away from the INM in the LMN-Y59C background compared with $L M N-W T\left(\log _{2}\right.$ [EMR-1/GFP] ratio $>1$ or $>3$, or else $<-1$ or $<-3$ for $L M N-Y 59 C-L M N$ $W T$ ). For these $L M N-Y 59 C$-sensitive sequences, we observed a striking reversal of the $L M N-Y 59 C$-induced change upon cec-4 ablation (Fig. 6C,D). The bins showing enhanced EMR-contact in LMN-Y59C (blue) were systematically shifted inwards in the double mutant (dark red), restoring the position that was scored in cec- $4 \Delta$ alone (Fig. 6C). The converse was also true (bins that shifted inward in the $L M N-Y 59 C$ mutant, were restored to the INM by cec-4 ablation; Fig. 6D). This cec- $4 \Delta$ rescue of aberrant chromatin architecture induced by $L M N-Y 59 C$ was not dependent on the size of the sequence window used; the effect was also clear in 100-kb bins along the left (pairing) arm of chromosome III (Fig. 6E).
Given the partial phenotype suppression by the CEC-4 Y87A/Y111A chromodomain mutant and the fact that chromosomal arms shifted more than the chromosomal cores (Supplemental Fig. S6A,B), we asked whether cec$4 \Delta$ preferentially acted on repetitive sequences or domains enriched for $\mathrm{H} 3 \mathrm{~K} 9 \mathrm{me} 2$ or $\mathrm{H} 3 \mathrm{~K} 9 \mathrm{me} 3$. We were able to score a marginal enrichment for repeat elements, but not for H3K9 methylation (Supplemental Fig. S6E,F). This may reflect limitations in sensitivity, because H3K9me was mapped in whole larvae (on mixed tissues), in which muscle is a minor fraction, while the EMRDamID changes are muscle-specific. Nonetheless, it suggests that genes and promoters, rather than repeat elements per se, respond to $L M N-Y 59 C$ and cec-4D.

Importantly, the effect of cec-4 ablation was more pronounced in the $L M N-Y 59 C$ mutant than in the $L M N$ WT background: While $12.3 \%$ of sequence bins were released from the periphery in the $L M N-W T$; cec- $4 \Delta$ double mutant, twice as many shifted in the $L M N-Y 59 C$; cec-4D strain (24.8\%) (Supplemental Fig. S6A,B). Indeed, most chromatin showed no change in position in the $L M N$ WT background upon cec-4 deletion, and surprisingly, if position changed, more shifted toward than away from the INM. The opposite was true in the LMN-Y59C strain. This suggests that the aberrant positioning of endogenous chromatin triggered by $L M N-Y 59 C$ might actually be mediated by CEC-4, given that cec- $4 \Delta$ is able to reverse the effects.

Once again, we examined whether transcription factors were enriched on the promoters of genes that shifted position in the $L M N-Y 59 C$; cec-4 $\Delta$ double mutant. We plotted the relative $\log _{2}$ (EMR-1/GFP) ratio of genes bearing E2F consensus motifs (either EFL-2 or EFL-1 and EFL-2), in $L M N-Y 59 C$ strains with or without cec-4. E2F motif-containing genes that were peripheral in LMN-Y59C shifted to a more central position in $c e c-4 \Delta$, and vice versa (Supplemental Fig. S7A). If we performed the analysis the other way around, and searched for transcription factor motif enrichment in the cec-4 sensitive sequences, we again identified the consensus for EFL-2, but not for EFL-1, as being strongly enriched (Supplemental Fig. S7B). This may indicate a special role for EFL-2 in nuclear organization in muscle. Of note, $44 \%$ of all muscle-expressed genes contain E2F motifs in their promoters (Supplemental Fig. S7C).

In summary, we found that EMR-DamID signals that change in the $L M N-Y 59 C$ mutant are efficiently reverted upon cec-4 ablation (Fig. 6). We conclude that cec-4 $\Delta$ compensates for gain-of-function effects of $L M N-Y 59 C$ with respect to global genome architecture. The genes and promoters most significantly affected are enriched for the motif recognized by EFL-2, an Rb-binding E2F homolog, or else for a nuclear receptor transcription factor, NHR66 (Supplemental Fig. S7B).

\section{Altered transcription in a lamin mutant can be reversed by cec-4 deletion}

We next examined transcriptional changes in the $L M N$ Y59C worms and asked whether they were rescued by 
Downloaded from genesdev.cshlp.org on April 26, 2023 - Published by Cold Spring Harbor Laboratory Press

LAD misorganization and its suppression in EDMD

A

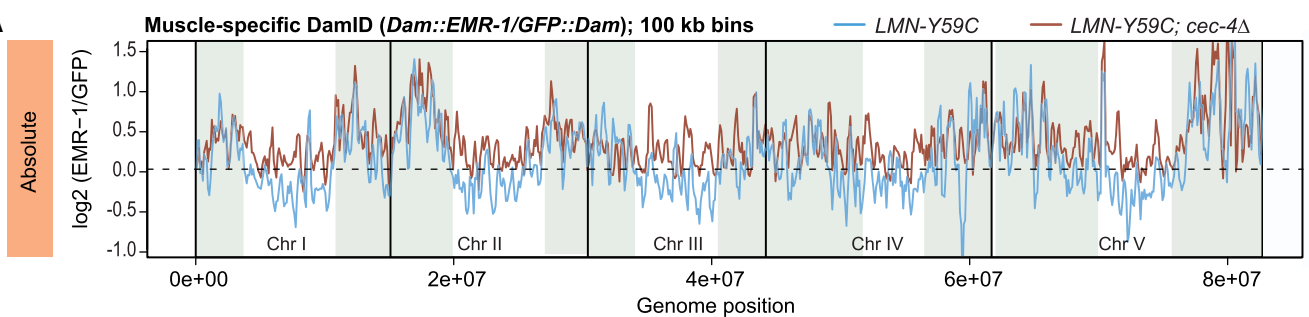

B

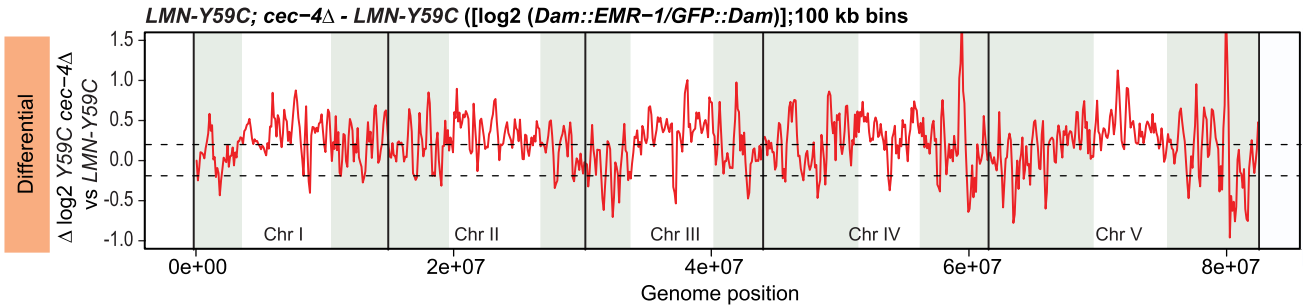

C

$10 \mathrm{~kb}$ bins with $\log 2$ enrichmen LMN-Y59C - LMN-WT
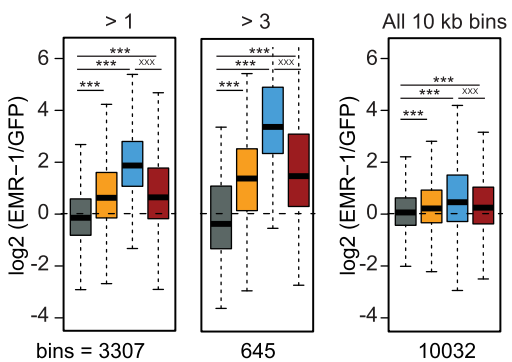

D $10 \mathrm{~kb}$ bins with log2 enrichment LMN-Y59C-LMN-WT
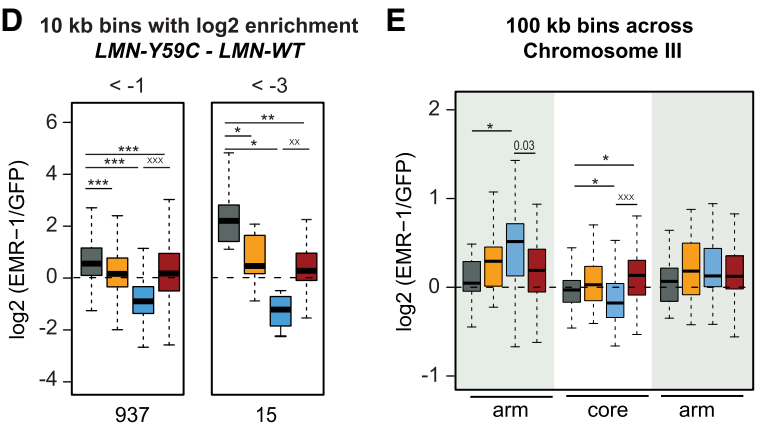

$\square L M N-W T \quad \square L M N-W T$; cec-4A $\square L M N-Y 59 C \quad \square \quad L M N-Y 59 C ;$ cec-4A

Figure 6. $L M N-Y 59 C$-induced EMR-DamID phenotypes in muscle are suppressed by cec-4 $\Delta$. (A) Line plot of $\log _{2}$ of EMR-1-Dam normalized to GFP-Dam from LMN-Y59C (blue) and LMN-Y59C; cec-4D (dark red) C. elegans L3/L4 muscle cells. Signal is averaged over three sliding 100-kb windows. Chromosome arms are shaded (see the Materials and Methods); dotted line indicates zero $\log _{2}$ ratio. $(B)$ Line plot of $E M R-1 / G F P\left(\log _{2}\right)$ of differences between $L M N-Y 59 C$; cec-4 4 versus $L M N-Y 59 C$. The dotted line indicates 0.2 and $-0.2 \log _{2}$ ratio thresholds. (C) Box plots comparing the frequency of contacts ( $\left.\log _{2}[\mathrm{EMR}-1 / \mathrm{GFP}]\right)$ for 10-kb bins that are enriched for $\log _{2}(L M N-Y 59 C)-\log _{2}(L M N$ -

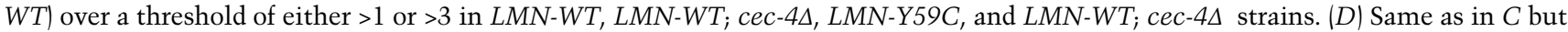
comparing the frequency of contacts ( $\log _{2}$ [EMR-1/GFP]) of 10-kb bins depleted for $\log _{2}(L M N-Y 59 C)-\log _{2}(L M N-W T)$ below a threshold of either $<-1$ or $<-3$ in $L M N-W T$ and $L M N-Y 59 C$ strains. (E) Box plot comparing the frequency of EMR-Dam contacts along chromosome III normalized to GFP-Dam in the indicated strains. Arm versus core designation and averaging as in A. Probability values from Wilcoxon rank sum tests are indicated: LMN-WT (Dam::EMR-1/GFP::Dam; GW1480/GW1481), LMN-WT; cec-4A (Dam::EMR-1/GFP::Dam; GW1482/GW1483), LMN-Y59C (Dam::EMR-1/GFP::Dam; GW1508/GW1485), and LMN-Y59C; cec-4D (Dam::EMR-1/GFP::Dam; GW1486/GW1487). $\left(^{*}\right) P<0.01 ;(* *) P<0.0001 ;(* * *) P<10^{-10}$, when compared with $L M N-W T$. Significance level compared with $L M N$ Y59C mutants $(\mathrm{x})$, are also given.

cec-4 deletion. We performed RNA-seq on whole worm L1 larvae in wild-type, cec-4D, LMN-Y59C and the $L M N$ Y59C; cec-4 $\Delta$ double-mutant strains (Fig. 7), determining significant transcript level changes by edgeR with an FDR $<0.01$ and FC $>1.5$ (Materials and Methods). The transcriptional changes in $L M N-Y 59 C$ and in the double mutant, while not dramatic, were reproducible between replicate sample pairs (Fig. 7A), allowing us to identify 187 genes with $>1.5$-fold change in transcript levels in each replica of the pairwise comparisons indicated in the heat map legend (Fig. 7B). There were not many transcript changes meeting this stringent criteria, 49 genes were up-regulated and 48 genes down-regulated $>1.5$-fold in the comparison of $L M N-Y 59 C$ versus WT L1 larvae (Supplemental Tables S1-S4). Importantly, and consistent with a previous report on cec-4 $\Delta$ embryos (Gonzalez-Sandoval et al. 2015), we found that the cec-4 ablation alone provoked very little change in steady state transcript abundance over WT L1 larvae (Fig. 7A), with the striking exception of a gene encoding a transmembrane G-coupled receptor, swr-85 (see also Gonzalez-Sandoval et al. 2015). Nonetheless, a large fraction of the transcripts whose levels were altered in the $L M N-Y 59 C$ mutant now showed sensitivity to the deletion of cec-4, with mRNA levels shifting in the opposite direction from that scored in the $L M N-Y 59 C$ mutant. That is, $\sim 30 \%$ of the genes shown in Figure $7 \mathrm{~B}$ as transcripts that changed significantly upon $L M N-Y 59 C$ expression showed compensatory effects in the $L M N-Y 59 C$; cec-4 $\Delta$ double mutant. In some cases the $L M N-Y 59 C$ led to down-regulation, and cec-4 $\Delta$ 
Harr et al.

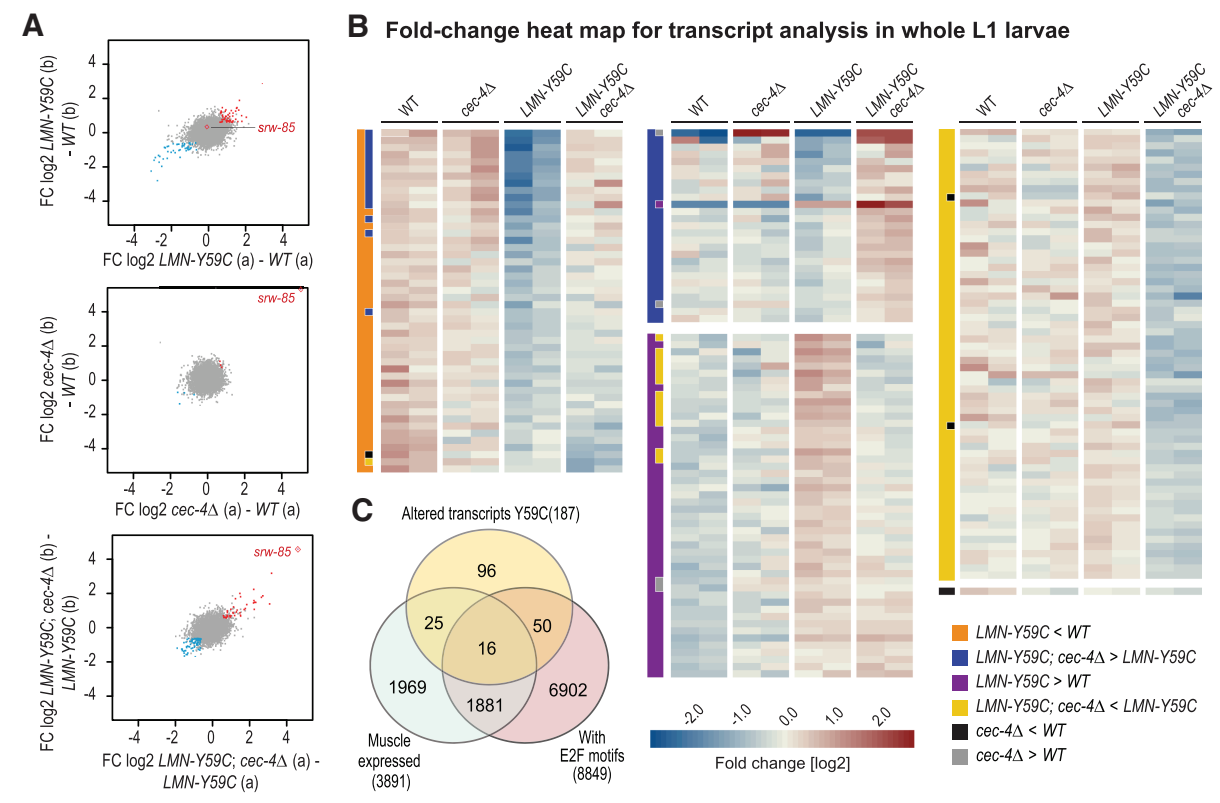

D Model for LMN-Y59C and CEC-4 engagement with Rb-E2F

Balanced interaction with CEC-4 and limited RbEFL-2-lamin interaction

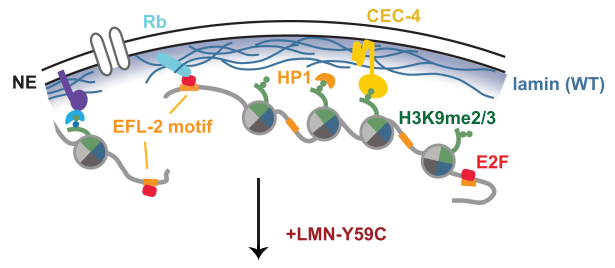

Lamin-Y59C induced chromatin hyper-sequestration

Enhanced anchoring through LMN-Y59C and CEC-4; Rb-EFL-2 may be enhanced

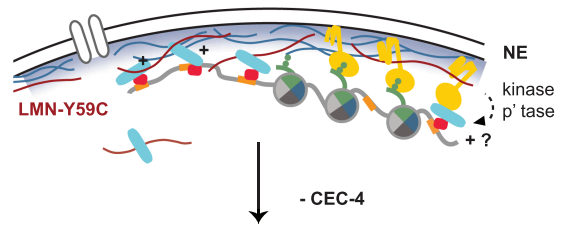

Loss of CEC-4 blocks hyper-sequestration

Loss of CEC-4 eliminates enhanced LMN-Y59C interaction, possible drop in Rb-EFL-2 occupancy

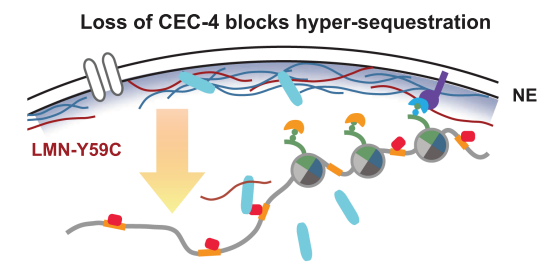

Figure 7. RNA-seq shows compensatory effects of cec-4D on $L M N$-Y59C-induced transcriptional changes. $(A)$ Scatter plots visualizing the replicate concordance of $\log _{2}$ fold changes (FC) in differential transcript abundances contrasting the two replicas (shown in $\left.A, B\right)$. (Top) LMN-Y59C (GW653) compared with wild type (WT, N2). (Middle) cec-4A (GW828) compared with WT. (Bottom) LMN-Y59C; cec-4 $(\mathrm{GW} 1468)$ compared with $L M N-Y 59 C$ (GW653). (Blue) Differential genes $(P<0.01)$ down in both replica pairs $\left[<-\log _{2}(1.5 \times)\right] ;($ red) differential genes $(P<0.01)$ up in both $\left[>\log _{2}(1.5 \times)\right]$. (Red open diamond) srw-85, a gene up-regulated in cec-4 $\Delta$ embryos (Gonzalez-Sandoval et al. 2015). (B) Heat map visualizing relative transcript abundances in L1 whole larval RNA in two replicates of each strain (see $A$ ). Each row represents a gene identified as differential by edgeR (FDR $<0.01$ and FC $>1.5$ over the indicated comparison based on average values). The two-column bar at the left of each gene set indicates the comparisons used to define the given gene set. Each subset is sorted by the FC between "LMN-Y59C" over "LMN-Y59C; cec4D" (last four columns). The color scale in the heat map indicates the FC deviation from the gene-wise average transcript abundance over all samples. $(C)$ Venn diagram of altered transcripts in mutant LMN-Y59C L1 larvae, muscleexpressed genes (Blazie et al. 2015), and genes with putative E2F sites (EFL-1 and EFL-2 pooled). (D) A model for LMN-Y59C and CEC-4 interaction with possible role for Rb-EFL-2. The top sketch indicates the INM components and chromatin components relevant for sequence positioning in differentiated muscle of C. elegans. In purple is a hypothesized unknown anchor. (Middle and bottom) The gainof-function LMN-Y59C mutation confers enhanced Rb-EFL-2 and/or enhanced CEC-4 binding, and the loss of CEC-4 counteracts this. ( $\mathrm{p}^{\prime}$ tase) Phosphatase. 
raised expression levels; in others, $L M N$-Y59C induced expression that $c e c-4 \Delta$ then reversed.

We examined the prominent $L M N$-Y59C-sensitive genes for functional pathways or relevant GO terms; $22 \%(41 / 187)$ of the $L M N-Y 59 C$-sensitive genes are muscle expressed, as defined by Blazie et al. (2015). This is not a strong enrichment, but given that RNA-seq was carried out in whole larvae, where muscle cells comprise only $10 \%$ of the somatic tissues, changes in low to moderately expressed muscle genes may fall below our limit of detection. There was no significant enrichment for ELF-2 motifs among the sequences or genes that change level in $L M N$-Y59C expressing larvae, although $27 \%$ of all ELF- 2 motif-containing promoters are found upstream of muscle-expressed genes. If we broadened the range to include both EFL-1 and EFL-2 motifs, we found that 35\% of the LMN-Y59C misregulated genes and 49\% of muscle-expressed genes bear such motifs within the genomic range of $-1.5 \mathrm{~kb}$ upstream of to $+0.5 \mathrm{~kb}$ downstream from the annotated transcription start sites (Fig. 7C). Thus, even without a statistically compelling link between the $L M N-Y 59 C$ induced changes in position and changes in expression, both assays identify EFL-2-or potentially an indirect effect on a complex containing EFL-1-as relevant downstream factors. Importantly, both E2F homologs bind $\mathrm{Rb}$, a repressor that was shown to bind mammalian Lamin A.

\section{Discussion}

Lamin A is a highly conserved coiled-coil intermediate filament protein of the nuclear lamina meshwork found adjacent to the nuclear envelope. Lamins are present in all eukaryotes that have an open mitosis, for the presence of at least one lamin family member is necessary to ensure proper nuclear reassembly after telophase (Nigg 1992). DamID data implicate lamins in the distribution of genomic domains bearing specific heterochromatic marks, most notably histone H3K9me2/3 (Kind et al. 2013; Solovei et al. 2013), even though lamin itself does not have a domain that can selectively bind silent chromatin. Indeed, the molecular recognition of chromatin by the nuclear lamina is still poorly defined in vertebrates (Bank and Gruenbaum 2011b; Dittmer and Misteli 2011; Camozzi et al. 2014).

Compromised integrity of the nuclear lamina leads to tissue-specific pathologies in a range of human diseases called laminopathies, some with severe pathologies, such as EDMD (Worman and Bonne 2007). We previously showed that the expression of a mutant form of C. elegans lamin (LMN-Y59C) generates a number of EDMD phenotypes in worms, mimicking the human disease-linked gain-of-function mutation, LMNA-Y45C. Not only did LMN-Y59C-expressing worms show perturbed body muscle function and compromised muscle ultrastructure, but the mutant lamin interfered with the relocation of an activated muscle-specific promoter away from the INM to the nuclear interior in adult muscle cells (Mattout et al. 2011). In most laminopathy studies, links that are proposed to exist between chromatin misorganization and the physiological dysfunction of muscle have remained on the level of correlation (Bank and Gruenbaum 2011b; Camozzi et al. 2014; Perovanovic et al. 2016), as no clear mechanisms were identified that linked chromatin misorganization to the disease.

Here we showed by means of muscle-specific emerinDamID that the worm mutation equivalent to the human LMNA Y45C mutation (LMN-Y59C) changes INM-chromatin interactions and genome architecture in C. elegans muscle nuclei. The $L M N-Y 59 C$ effect is dominant, given that the mutant contributes at most $20 \%$ of the endogenous wild-type lamin complement, yet the phenotypes are reproducibly manifest. Whereas the vast majority $(>90 \%)$ of the genome does not appear to shift position upon $L M N$-Y59C expression, we found that $\sim 4 \%$ of sequences genome-wide shift to the nuclear periphery, while $\sim 4 \%$ become more central. Most of the sequences that become peripheral are enriched on the heterochromatin-rich autosomal arms (Supplemental Fig. S6A,B), yet there is no statistical enrichment of $\mathrm{H} 3 \mathrm{~K} 9$ methylation on these sequences (Supplemental Fig. S1E,F). Therefore, we were prompted to look for transcription factor enrichment on the mislocalized chromatin. This revealed a selective enrichment for the binding consensus of the E2F homologue, EFL-2 (Supplemental Fig. S2A).

The E2F family of transcription factors is regulated by the formation of an inhibitory complex with $\mathrm{Rb}$, a key tumor suppressor in mammals. As an activator, E2F triggers key cell cycle events, including apoptosis, and controls differentiation in certain tissues. This was particularly well-characterized for the C. elegans DREAM complex, which contains a E2F-DP heterodimer (EFL-1 and DPL1), a retinoblastoma-like pocket protein (LIN-35), and a five-subunit MuvB complex (LIN-9, LIN-37, LIN-52, LIN-53, and a DNA-binding subunit, LIN-54) (Ceol and Horvitz 2001; Petrella et al. 2011; Kudron et al. 2013; Latorre et al. 2015; Goetsch et al. 2017). E2F-binding sites are found throughout the C. elegans genome, and the binding of the worm E2F homologs, EFL-1 or EFL-2, can lead to both up-regulation and down-regulation of gene expression, although the loss of $\mathrm{E} 2 \mathrm{~F}$ or $\mathrm{Rb}$ alone does not necessarily alter gene expression (Petrella et al. 2011; Latorre et al. 2015; Goetsch et al. 2017). In LMN-Y59C-affected sequences, we found a specific enrichment for a smaller motif, that of EFL-2, which lacks the additional LIN-54 recognition motif. Thus, we propose a role of EFL-2, and possibly the EFL-2/Rb complex, in muscle nuclear organization that is distinct from the activities of the DREAM complex (Ceol and Horvitz 2001). In emerindeficient mice the misregulation of E2F by $\mathrm{Rb}$ has been implicated in muscle-related pathologies (Melcon et al. 2006), and lamin A mutants were shown to impair muscle differentiation in vitro in an $\mathrm{Rb}$-dependent manner (Favreau et al. 2004; Markiewicz et al. 2005). While suggestive, these earlier studies did not explore links to chromatin organization, even though $\mathrm{Rb}$ had been reported to bind the nuclear lamina.

We show here that the changes in the spatial organization of the genome likely cause the muscle laminopathy linked to $L M N-Y 59 C$ (Y45C in human $L M N A$ ), because 
we are able to counteract the $L M N$-Y59C-induced phenotypes by deleting the C. elegans heterochromatin binding protein, CEC-4. CEC-4 is a H3K9methyl-binding chromodomain protein that is associated with the nuclear lamina (Gonzalez-Sandoval et al. 2015). It also has a large disordered C-terminal domain of unknown function that is necessary for proper INM localization. Ablation of cec-4 releases both heterochromatic arrays and endogenous heterochromatin from the nuclear periphery in embryos (Gonzalez-Sandoval et al. 2015) and triggers reporter array expression in muscle cells (Fig. 2). CEC-4 contributes actively to the positioning of endogenous sequences in muscle cells (Fig. 6), and its ablation largely reverses the changes in chromatin distribution brought about by laminopathy mutant $L M N-Y 59 C$. Intriguingly, the binding motif of the E2F-homolog EFL-2, is enriched in the sequences that shift position in the $L M N-Y 59 C$ mutant. With respect to gene expression, changes provoked by the $L M N-Y 59 C$ mutation are, in some cases, reversed by cec- $4 \Delta$, arguing that CEC-4 is a downstream effector of the pathology-provoking lamin variant (Fig. 7A; Supplemental Fig. S7A). Whether CEC-4 interacts with, or modulates EFL-2 function remains to be explored.

There are at least two hypotheses that could explain these observations. There may be a gain (or, respectively, loss) of H3K9 methylation in the LMN-Y59C mutant that leads to chromatin relocation by altering interaction with CEC-4. Alternatively, CEC-4 may have an alternative function in the $L M N-Y 59 C$-expressing strain that interferes with the effect of the lamin mutation on gene or locus positioning (Fig. 7D). The fact that chromatin domains shift both toward and away from the INM in the $L M N-Y 59 C$ mutant, and that cec-4 deletion compensates for both, argues that CEC-4 may have more than one mode of action. Nonetheless, there are roughly threefold as many highly significant shifts (difference $\log _{2}>3$ ) toward the nuclear periphery in the $L M N-Y 59 C$ mutant as away from it $\left(\log _{2}<-3\right)$. Given that the loss of cec-4 suppresses not only aspects of chromatin positioning, but other muscle-relevant $L M N$-Y59C-induced defects, the double mutant provides a strong argument that the muscle pathology correlated with $L M N-Y 59 \mathrm{C}$ stems from, and not only correlates with, altered patterns of chromatin organization.

We performed whole worm RNA-sequencing on L1 larvae to monitor transcriptional changes in $L M N-Y 59 C$ and their suppression by cec- $4 \Delta$. Around 100 genes showed significant up-regulation or down-regulation upon expression of the $L M N-Y 59 C$ mutant, and by combining cec-4 $\Delta$ with $L M N-Y 59 C$, we could compensate or reverse a large fraction of these (Fig. 7; see Supplemental Table S1-S4). Consistent with earlier work in embryos (Gonzalez-Sandoval et al. 2015), we found few transcriptional differences provoked by cec- $4 \Delta$ alone (Fig. 7). Nonetheless, it is unclear which of the affected genes may have relevance for the observed muscle pathology. Roughly $22 \%$ of the transcripts altered are muscle-expressed (Fig. 7C), and 17 out of the 41 genes that are misregulated by $L M N-Y 59 C$ and counteracted by cec-4 4 , harbor E2F sites. While striking, the percentage of muscle-expressed genes bearing E2F sites is even higher $(48 \%)$. Once again, in the affected sequences, the motif for the less common E2F homolog, EFL-2, was far more enriched than that of the more common E2F homolog, EFL-1 (Supplemental Fig. S7C). Thus, EFL-2 may have a unique role in muscle homeostasis (Narasimhan et al. 2015).

We propose that at least part of the $L M N-Y 59 C$-sensitive genes depend on $\mathrm{E} 2 \mathrm{~F}$ and $\mathrm{Rb}$ for subnuclear positioning, and possibly also for transcriptional regulation, although the two do not strictly correlate. Unfortunately, in worms, $\mathrm{Rb}$ and EFL-1 deletions have many other pleiotropic effects on development, including sterility and embryonic lethality, rendering their roles difficult to test (Petrella et al. 2011; Kudron et al. 2013; Goetsch et al. 2017). EFL-2 is less well studied, but eff-2 RNAi did not mimic loss of efl-1 (Ceol and Horvitz 2001). Whereas the CEC-4 CDY2A mutant, which ablates CEC-4 binding to H3K9me (Gonzalez-Sandoval et al. 2015), partially suppresses array mislocalization and head-thrashing defects, we do not rule out that CEC-4 might interact with other factors through its unstructured C-terminal domain. These factors may be histone acetyltransferases or deacetylases, kinases or phosphatases, or transcription factors themselves. Preliminary pull-down assays with a tagged version on CEC-4 identified only the expected INM/lamina components as strong interactors. Nonetheless, other interactions are likely to occur, and may alter chromatinlamina interactions.

We searched the literature for the mammalian equivalent of CEC-4, especially with respect to chromatin interaction with the nuclear lamina. Based on functional and structural homology, the mammalian proline-rich 14 protein (PRR14) has been proposed to represent a CEC-4 functional homolog in that it is associated with $\operatorname{lamin} \mathrm{A} / \mathrm{C}$ and tethers heterochromatin to the INM during interphase and mitotic exit (Poleshko et al. 2013). The ablation of PRR14 impeded muscle differentiation, while its overexpression enhanced muscle differentiation in vitro /Yang and Yuan 2015), apparently by enhancing the activity of MyoD. Unlike CEC-4, PRR14 can bind HP1a, and thus the differentiation effects were thought to be due to a loss of heterochromatin stabilization. No genetic interaction with lamin A/C nor synergy with laminopathic mutations has been reported for prr14 knockdown, thus its relationship to our study remains unclear. Unlike PRR14, CEC-4 appears to actually compete with HP-1 for binding H3K9 methylated chromatin (Gonzalez-Sandoval et al. 2015).

With respect to the LMNA-Y45/LMN-Y59C allele, we note that this mutation is in one of the most highly conserved $\alpha$-helical domains of lamin $\mathrm{A} / \mathrm{C}$, and that a cytosine replaces a key hydrophobic contact in the heptad repeat of the 1B coiled-coil domain. The LMN-Y59C lamin forms filaments in vitro, and when expressed in worms, it forms a perinuclear ring together with wild-type lamin. However, consistent with the prediction that the dimers might be less stable, FRAP data confirms a more rapid turnover of LMN-Y59C within the lamina (Wiesel et al. 2008; Mattout et al. 2011). Lamin A, like LMN-1 in worms, functions both at the INM and in the nucleoplasm, and 
through its affinity for $\mathrm{Rb}$, it may activate or repress genes regulated by $\mathrm{E} 2 \mathrm{~F}$. The $1 \mathrm{~B}$ coiled-coil domain itself is unlikely to alter $\mathrm{Rb}$ interaction, as $\mathrm{Rb}$ binding was mapped to aa 247-355 in lamin A (Ozaki et al. 1994). However, a more rapid turnover of the LMN-Y59C dimer could change the distribution of $\mathrm{Rb}$-bound promoters, releasing some sites, while allowing others to be sequestered. Those sequences showing NE-sequestration, like the heterochromatic array, are released upon cec-4 ablation. For this reason we also entertain the model that CEC-4 may promote Rb-EFL-2 stability (Fig. 7D). A failure to release and activate tissue-specific promoters in a muscle-specific manner may also be relevant for human patients carrying the LMN-Y45C mutation (Melcon et al. 2006; Camozzi et al. 2014).

In summary, here we provided novel evidence for a genetic suppressor of an EDMD-like disease provoked by a dominant missense mutation in lamin A, and its suppression by loss of a CD protein with specificity for histone H3K9me, i.e., CEC-4. The simplest interpretation is that this deletion compensates for aberrant nuclear positioning of crucial sequences at the INM. We confirmed this for muscle using muscle-specific DamID. This mechanism of suppression is consistent with major hypotheses concerning the effects of lamin mutations on tissue integrity (Worman and Bonne 2007; Bank and Gruenbaum 2011a; Camozzi et al. 2014) and argues that specific lamin A mutations have both gain-of-function activity (generating promiscuous LADs) and dominant-negative activity (an inability to initiate and spread LADs), which has consequences during the differentiation of cell fate (Perovanovic et al. 2016). The Perovanovic et al. (2016) study in mammalian cells also used DamID to map LADs in laminopathy mutants, and proposed that the diseases caused by certain lamin mutations resulted from epigenetic changes. They documented changes in histone $\mathrm{H} 3$ methylation and DNA methylation at a large number of sites. Our contribution goes one step further, in that we show that one can reverse the changes in genome organization (i.e., LADs) by the deletion of a specific NE protein that reads H3K9 methylation. With this, we have substantiated the hypothesis that chromatin domain organization is a crucial player in laminopathic disease. Our study suggests that antagonizing such phenomena may open therapeutic options for treating laminopathies.

\section{Materials and methods}

Strains and crosses

C. elegans strains (Supplemental Table S5) were maintained and manipulated as previously described (Brenner 1974). Strains were maintained at $22.5^{\circ} \mathrm{C}$ for all studies unless otherwise noted. Worms were grown on NGM plates and fed OP50 bacteria except for emerin (EMR-1)-DamID experiments.

\section{Head thrashing assay}

To score C. elegans motility, L4 staged worms were gently transferred by pick to $30 \mu \mathrm{L}$ of nuclease-free water (Qiagen) in an 18well uncoated slide (Ibidi 81821) with two worms/well. Fifteen minutes after the last worm was transfered, the number of head movements to the right per $30 \mathrm{sec}$ was counted under a bright light microscope. Room temperature was monitored closely and maintained within $21^{\circ} \mathrm{C}-23^{\circ} \mathrm{C}$. Worms for each experiment were processed and analyzed in parallel as small temperature changes alter locomotion, by a single-blind observer. No more than three strains were analyzed in parallel to ensure consistency. Worm health was verified by touch response to the pick; unreactive worms were excluded.

RNAi

RNAi experiments were performed by placing L1 larvae on feeding plates as described previously (Timmons et al. 2001). The progeny of the RNAi fed C. elegans were scored for phenotypes. Sequence specificity of RNAi clones used were sequenced for confirmation. As a mock RNAi control, the L4440 vector (Fire vector library) was modified by removing an $E c o R V$ fragment containing 25 bp identical to GFP-LacI.

\section{Staining}

For phalloidin staining, C. elegans were synchronized by bleaching adults and seeding L1 larvae after hatching overnight. L1 larvae were collected at the young adult stage (before eggs start to form). For all centrifugation steps, a swinging-bucket rotor was used at $800 \mathrm{~g}$ and at room temperature. Worms were washed twice in $\mathrm{M} 9$ and transferred to a low-bind $1.5-\mathrm{mL}$ tube. Following fixation with $4 \%$ paraformaldehyde (EMS Cat\#15710) in $0.1 \mathrm{M}$ $\mathrm{Na} 2 \mathrm{HPO} 4$ for $15 \mathrm{~min}$ with rotation, worms were pelleted, and then permeabilized with $500 \mu \mathrm{L}$ of cold acetone $\left(100 \%\right.$ at $-20^{\circ}$ C) for $5 \mathrm{~min}$ at $-20^{\circ} \mathrm{C}$. Worms were pelleted and washed by rotation in PBS containing $0.5 \%$ Triton X-100 and $30 \mathrm{mM}$ glycine (PBS-TG) for $10 \mathrm{~min}$. Staining was with $500 \mu \mathrm{L}$ of tetramethylrhodamine-phalloidin (1:250; Molecular Probes R415) in PBS-TG for $30 \mathrm{~min}$, rotating at room temperature in the dark. Following centrifugation and three washes with PBS-TG at room temperature, they were left to settle on poly-L-lysine-coated slides for 5 min without drying. Excess liquid was removed and mounting medium (ProLong Gold antifade mountant, Thermo Scientific P36930) was added. The monitoring of defective sarcomeres was performed by a single-blind observer, with partial doubleblind checking by an independent assistant.

\section{Microscopy}

Microscopy was carried out on spinning-disk multipoint confocal microscopes (AxioImager M1 [Carl Zeiss]+Yokogawa CSU-22 scan head, Plan-Neofluar 100×/1.45 NA oil objective, EM-CCD camera [Cascade II, Photometrics], and MetaMorph 7.7.2 software or Olympus IX81 + Yokogawa CSU-X1 scan head, PlanApo $100 \times / 1.45$ TIRFM objective, $2 \times$ back-illuminated EM-CCD EvolveDelta (Photometrics), and VisiView software GFP) and mCherry fluorophores were excited using a Toptica iBeamSmart 491-nm and 561-nm lasers. For live microscopy, plates were washed twice with M9 to remove adults and larvae and leave only eggs on the bacteria layer. L1 larvae hatched during 2-3 h and were collected by M9 wash. For live imaging, animals were mounted on slides coated with $2 \%$ agarose pads, supplemented with $0.1 \% \mathrm{NaN}_{3}$ and $1 \mathrm{mM}$ levamisole. For early adult live microscopy, worms were synchronized by bleaching gravid adults, and seeding L1 larvae after hatching overnight. Once worms reached early adult stage (before formation of eggs) they were washed twice in M9 and mounted on coverslips in the presence of levamisole for imaging. 
Harr et al.

\section{Image analysis}

Single- or multiplane (maximum intensity $\mathrm{Z}$ projections) images and analysis were generated using Fiji/ImageJ software 6. Quantitation of gwIs 4 and cals 3 reporter distribution on focal stacks of images was done with plug-in PointPicker (http://bigwww.epfl .ch/thevenaz/pointpicker) as described previously (Meister et al. 2010a). For high-throughput image analysis, 3D stacks were processed and analyzed as described previously (Gonzalez-Sandoval et al. 2015; Hauer et al. 2017). For statistical analysis and data representation, only nuclei with two foci were taken into account. Nuclei touching the image boarders at the $X_{-}, Y_{-}$, or $Z$-axis were excluded from the analysis. Table operations, data filtering (two foci per nucleus), and normalization were performed with KNIME (Dietz and Berthold 2016). The control (WT) condition was set to 1 and data derived from mutants or other experimental conditions are shown relative to the control. Distributions were plotted with $\mathrm{R}$ as violin plot graphs.

\section{Muscle-specific emerin (EMR-1) DamID}

DamID was performed and analyzed largely as described in Gómez-Saldivar et al. (2016) and Cabianca et al. (2019). In this study, three biological replicas were prepared and all strains were grown in parallel in each replica and for all strains the analysis was performed in biological triplicates. C. elegans strains were synchronized by seeding four 10-cm NGM plates with E. coli GM119 Dam bacteria with $\sim 4000 \mathrm{~L} 1 \mathrm{~s}$ per plate. Animals were incubated for $26 \mathrm{~h}$ at $22.5^{\circ} \mathrm{C}$ until L3/L4 stage, before collection for DamID procedure. The triplicates were prepared and sequenced separately. Individual samples within each replica were barcoded, pooled, and then sequenced on a HiSeq 2500 (Illumina) sequencer with 50-cycle single-end reads. Most analyses were performed in R Studio (version 1.0.153) using the damid.seq.R pipeline (Sharma et al. 2016; available at http://www.github.com/damidseq/ RDamIDSeq). This pipeline identifies and maps specifically those NGS reads that contain the DamID adapters (adapt.seq= "CGCGGCCGAG") and corresponds to genomic fragments flanked by GATC sites (restr.seq = "GATC"). Mapping was performed to the C. elegans genome release WBCel235 (species= "BSgenome.Celegans.UCSC.ce11"). The pipeline provides absolute and relative read counts both per GATC fragment and per user-defined bin size. The number of reads obtained for each replicate in each strain ranged from 35,000 to 32.3 million (specifically, one was $\sim 35,000$ and all others were $>200,000$ ), but all were well above the level required for robust results $(10,000$ reads $)$ for 10-kb DamID resolution (Sharma et al. 2016). The pipeline computed the $\log _{2}$ ratio between relative Dam::EMR-1 and GFP::Dam reads within each replica and the Spearman correlation coefficients calculated pairwise between replicas ranged from 0.56 to 0.99 (median $=0.93$ ). Worm chromosomes have no repeat-rich centromere, and repeat elements are enriched on chromosome arms. It is therefore standard to compare chromosome arms versus cores for LAD behavior (Ikegami et al. 2010). Following standard practice, we used the following border coordinates: ChrI 3745632 and 10809938, ChrII 4708341 and 11877168 , ChrIII 3508994 and 9947268, ChrIV 7317812 and 12176625, ChrV 8125434 and 13849337, and ChrX 41919362. Pairwise Wilcoxon rank test values were calculated in $\mathrm{R}$.

RNA-seq samples preparation

For RNA-seq, synchronized L1 larvae were obtained by bleaching gravid adults and the eggs recovered were left to hatch 16 $\mathrm{h}$ at room temperature in M9. The L1 larvae were refed for 2.5 $\mathrm{h}$, washed three times in M9, resuspended in $100 \mu \mathrm{L}$ of $\mathrm{M} 9$ and $400 \mu \mathrm{L}$ of Trizol (Ambion), and snap-frozen in $\mathrm{N}_{2}$ (liq) for RNA extraction. Extraction of RNA used four freeze-thaw cycles from N2(liq) to a $42^{\circ} \mathrm{C}$ heat bath, followed by the addition of $200 \mu \mathrm{L}$ of Trizol to each sample. Vigorous vortexing at room temperature (five cycles at 30 -sec vortex, $30 \mathrm{sec}$ on ice), was followed by $5 \mathrm{~min}$ at room temperature. RNA extraction was by $140 \mu \mathrm{L}$ of chloroform (15 sec of shaking and $2 \mathrm{~min}$ at room temperature); after centrifugation at $4^{\circ} \mathrm{C}$, the aqueous phases were transferred to fresh tubes. An equal volume of $70 \% \mathrm{EtOH}$ was added slowly and the homogeneous mixture was transferred to a Qiagen RNeasy spin column (RNeasy kit, Qiagen 74104), including a subsequent 30-min DNase treatment. Stranded library preparation was performed with a TruSeq Illumina mRNA-seq library preparation kit. The quality of the resulting libraries was assessed with an Agilent Bioanalyzer and concentrations were measured with a Qubit fluorometer prior to pooling. Fifty-nucleotide single-end sequencing was done on an Illumina HiSeq 2500.

\section{RNA-seq analysis}

For each genotype the RNA-seq data from two biological replicate samples were mapped to the C. elegans genome (ce10) using the $\mathrm{R}$ package QuasR v1.22.0. QuasR includes the short read aligner bowtie to consider only uniquely mapping reads. Count tables of reads mapping within annotated exons in WormBase (WS220) were normalize by division by the total number of reads in each library and multiplied by the average library size. Transformation into $\log _{2}$ space was performed after the addition of a pseudocount of eight in order to minimize large changes in abundance fold change (FC) caused by low count numbers. The edgeR package v3.24 was applied to select genes with differential transcript abundances between the indicated genotypes (contrasts) based on false discovery rates (FDR $<0.01)$ and fold changes $(\mathrm{FC} \geq 1.5)$, independently, in each of two replicate sample pairs. For each differentially expressed transcript (duplicate calls in different contrasts excluded) the average abundance over all samples was computed, and the FC deviations were represented in heat maps.

\section{Transcription factor motif analysis}

A compendium of transcription factor weight matrices (Narasimhan et al. 2015) was downloaded (http://hugheslab.ccbr.utoronto .ca/supplementary-data/CeMotifs) to examine the set in the file "TF_Information.txt." These were used to scan the C. elegans ce10 genome using the matchPWM function in the Bioconductor package Biostrings. Only hits with a minimum score of 10 were considered unless the maximum obtainable score by the weight matrix was $<10$. In that case, the maximum obtainable score was required. The resulting binding sites were then intersected with gene promoter annotation (WS220, $1500 \mathrm{bp} \mathrm{5}$, and $500 \mathrm{bp}$ $3^{\prime}$ of transcription start site) to determine the number of sites for each transcription factor and gene.

\section{Data availability}

RNA sequencing and EMR-1-DamID data are available through Gene Expression Omnibus (GEO), SuperSeries GSE136577 with SubSeries GSE136576 and GSE135850.

\section{Acknowledgments}

We dedicate this paper to our dear colleague and coauthor, Michael Hauer, who passed away during revisions. We thank the 
Caenorhabditis Genetics Center (CGC) funded by National Institutes of Health Office of Research Infrastructure Programs (P40 OD010440) for strains. We are grateful to P. Sartre for technical help and to Y. Gruenbaum, I. Katic, and members of the Friedrich Miescher Institute Genomics and Microscopy Facilities for advice and support. J.C.H. was supported by an FP7 Marie Curie Action Intra-European Fellowship and by a grant from the Foundation Suisse de Recherche sur les Maladies Musculaires. A.M. was funded by the same and by a Marie Heim-Vögtlin grant from the Swiss National Science Foundation. C.M.-J., R.R.-B., and P.A. thank the Spanish State Research Agency and the European Regional Development Fund (BFU2016-79313-P and MDM-20160687) for support. This project received funding from the European Research Council under the European Union's Horizon 2020 Research and Innovation program (Epiherigans grant no. 743312 to S.M.G.).

J.C.H. and S.M.G. wrote the manuscript. J.C.H. and A.M. designed and performed experiments and interpreted results. C.M.-J. and R.R.-B. performed DamID experiments and analysis. V.K. contributed to microscopy and motility experiments and invaluable technical support. A.G.-S. created strains for CEC-4(CD) rescue experiments. J.P. and C.D.S. performed bioinformatic analyses and interpreted results. M.H.H. did the high-throughput image analysis. P.A. analyzed DamID data, interpreted results, and provided funding. S.M.G. interpreted results, revised the paper, and provided funding.

\section{References}

Bank EM, Gruenbaum Y. 2011a. Caenorhabditis elegans as a model system for studying the nuclear lamina and laminopathic diseases. Nucleus 2: 350-357. doi:10.4161/nucl.2.5 .17838

Bank EM, Gruenbaum Y. 2011b. The nuclear lamina and heterochromatin: a complex relationship. Biochem Soc Trans 39: 1705-1709. doi:10.1042/BST20110603

Barkan R, Zahand AJ, Sharabi K, Lamm AT, Feinstein N, Haithcock E, Wilson KL, Liu J, Gruenbaum Y. 2012. Ce-emerin and LEM-2: essential roles in Caenorhabditis elegans development, muscle function, and mitosis. Mol Biol Cell 23: 543-552. doi:10.1091/mbc.e11-06-0505

Blazie SM, Babb C, Wilky H, Rawls A, Park JG, Mangone M. 2015. Comparative RNA-seq analysis reveals pervasive tissue-specific alternative polyadenylation in Caenorhabditis elegans intestine and muscles. BMC Biol 13: 4. doi:10.1186/s12915015-0116-6

Brenner S. 1974. The genetics of Caenorhabditis elegans. Genetics 77: 71-94.

Cabianca DS, Muñoz-Jiménez C, Kalck V, Gaidatzis D, Padeken J, Seeber A, Askjaer P, Gasser SM. 2019. Active chromatin marks drive spatial sequestration of heterochromatin in C. elegans nuclei. Nature 569: 734-739. doi:10.1038/s41586-0191243-y

Camozzi D, Capanni C, Cenni V, Mattioli E, Columbaro M, Squarzoni S, Lattanzi G. 2014. Diverse lamin-dependent mechanisms interact to control chromatin dynamics. Nucleus 5: 427-440. doi:10.4161/nucl.36289

Ceol CJ, Horvitz HR. 2001. Dpl-1 DP and efl-1 E2F act with lin-35 $\mathrm{Rb}$ to antagonize Ras signaling in C. elegans vulval development. Mol Cell 7: 461-473. doi:10.1016/S1097-2765(01) 00194-0

Cho S, Vashisth M, Abbas A, Majkut S, Vogel K, Xia Y, Ivanovska IL, Irianto J, Tewari M, Zhu K, et al. 2019. Mechanosensing by the lamina protects against nuclear rupture, DNA damage, and cell-cycle Arrest. Dev cell 49: 920-935.e5. doi:10.1016/j .devcel.2019.04.020

Cohen-Fix O, Askjaer P. 2017. Cell biology of the Caenorhabditis elegans nucleus. Genetics 205: 25-59. doi:10.1534/genetics .116 .197160

Dietz C, Berthold MR. 2016. KNIME for open-source bioimage analysis: a tutorial. Adv Anat Embryol Cell Biolpp 219: 179197. doi:10.1007/978-3-319-28549-8_7

Dittmer TA, Misteli T. 2011. The lamin protein family. Genome Biol 12: 222. doi:10.1186/gb-2011-12-5-222

Elenbaas JS, Bragazzi Cunha J, Azuero-Dajud R, Nelson B, Oral EA, Williams JA, Stewart CL, Omary MB. 2018. Lamin A/C maintains exocrine pancreas homeostasis by regulating stability of RB and activity of E2F. Gastroenterology 154: 1625-1629.e8. doi:10.1053/j.gastro.2018.01.024

Favreau C, Higuet D, Courvalin JC, Buendia B. 2004. Expression of a mutant lamin A that causes Emery-Dreifuss muscular dystrophy inhibits in vitro differentiation of C2C12 myoblasts. Mol Cell Biol 24: 1481-1492. doi:10.1128/MCB.24.4 .1481-1492.2004

Frock RL, Kudlow BA, Evans AM, Jameson SA, Hauschka SD, Kennedy BK. 2006. Lamin A/C and emerin are critical for skeletal muscle satellite cell differentiation. Genes Dev 20: 486500. doi:10.1101/gad.1364906

Goetsch PD, Garrigues JM, Strome S. 2017. Loss of the Caenorhabditis elegans pocket protein LIN-35 reveals MuvB's innate function as the repressor of DREAM target genes. PLoS Genet 13: e1007088. doi:10.1371/journal.pgen .1007088

Gómez-Saldivar G, Meister P, Askjaer P. 2016. DamID analysis of nuclear organization in Caenorhabditis elegans. Methods Mol Biol 1411: 341-358. doi:10.1007/978-1-49393530-7_22

González-Aguilera C, Ikegami K, Ayuso C, de Luis A, Íñiguez M, Cabello J, Lieb JD, Askjaer P. 2014. Genome-wide analysis links emerin to neuromuscular junction activity in Caenorhabditis elegans. Genome Biol 15: R21. doi:10.1186/gb2014-15-2-r21

Gonzalez-Sandoval A, Towbin Benjamin D, Kalck V, Cabianca Daphne S, Gaidatzis D, Hauer MH, Geng L, Wang L, Yang T, Wang X, et al. 2015. Perinuclear anchoring of H3K9methylated chromatin stabilizes induced cell fate in C. elegans embryos. Cell 163: 1333-1347. doi:10.1016/j.cell.2015 .10 .066

Harr JC, Gonzalez-Sandoval A, Gasser SM. 2016. Histones and histone modifications in perinuclear chromatin anchoring: from yeast to man. $E M B O$ Rep 17: 139-155. doi:10.15252/ embr.201541809

Hauer MH, Seeber A, Singh V, Thierry R, Sack R, Amitai A, Kryzhanovska M, Eglinger J, Holcman D, Owen-Hughes $\mathrm{T}$, et al. 2017. Histone degradation in response to DNA damage enhances chromatin dynamics and recombination rates. Nature Struct Mol Biol 24: 99-107. doi:10.1038/ nsmb.3347

Ikegami K, Egelhofer TA, Strome S, Lieb JD. 2010. Caenorhabditis elegans chromosome arms are anchored to the nuclear membrane via discontinuous association with LEM-2. Genome Biol 11: R120. doi:10.1186/gb-2010-1112-r120

Kind J, Pagie L, Ortabozkoyun H, Boyle S, de Vries SS, Janssen $\mathrm{H}$, Amendola M, Nolen LD, Bickmore WA, van Steensel B. 2013. Single-cell dynamics of genome-nuclear lamina interactions. Cell 153: 178-192. doi:10.1016/j.cell.2013.02 .028 
Kosak ST, Skok JA, Medina KL, Riblet R, Le Beau MM, Fisher AG, Singh H. 2002. Subnuclear compartmentalization of immunoglobulin loci during lymphocyte development. Science 296: 158-162. doi:10.1126/science.1068768

Kudron M, Niu W, Lu Z, Wang G, Gerstein M, Snyder M, Reinke V. 2013. Tissue-specific direct targets of Caenorhabditis elegans $\mathrm{Rb} / \mathrm{E} 2 \mathrm{~F}$ dictate distinct somatic and germline programs. Genome Biol 14: R5. doi:10.1186/gb-2013-14$1-\mathrm{r} 5$

Latorre I, Chesney MA, Garrigues JM, Stempor P, Appert A, Francesconi M, Strome S, Ahringer J. 2015. The DREAM complex promotes gene body H2A.Z for target repression. Genes DeV 29: 495-500. doi:10.1101/gad.255810.114

Liu T, Rechtsteiner A, Egelhofer TA, Vielle A, Latorre I, Cheung MS, Ercan S, Ikegami K, Jensen M, Kolasinska-Zwierz P, et al. 2011. Broad chromosomal domains of histone modification patterns in C. elegans. Genome Res 21: 227-236. doi:10 $.1101 /$ gr.115519.110

Lloyd DI, Trembath RC, Shackleton S. 2002. A novel interaction between lamin A and SREBP1: implications for partial lipodystrophy and other laminopathies. Human Mol Gen 11: 769-777. doi:10.1093/hmg/11.7.769

Markiewicz E, Dechat T, Foisner R, Quinlan RA, Hutchison CJ. 2002. Lamin A/C binding protein LAP2 $\alpha$ is required for nuclear anchorage of retinoblastoma protein. Mol Biol Cell 13: 4401-4413. doi:10.1091/mbc.e02-07-0450

Markiewicz E, Ledran M, Hutchison CJ. 2005. Remodelling of the nuclear lamina and nucleoskeleton is required for skeletal muscle differentiation in vitro. I Cell Sci 118: 409-420. doi:10.1242/jcs.01630

Mattout A, Pike Brietta L, Towbin Benjamin D, Bank Erin M, Gonzalez-Sandoval A, Stadler Michael B, Meister P, Gruenbaum Y, Gasser Susan M. 2011. An EDMD mutation in C. elegans lamin blocks muscle-specific gene relocation and compromises muscle integrity. Curr Biol 21: 1603-1614. doi:10.1016/j.cub.2011.08.030

Meister P, Gehlen LR, Varela E, Kalck V, Gasser SM. 2010a. Visualizing yeast chromosomes and nuclear architecture. Methods Enzymol 470: 535-567. doi:10.1016/S0076-6879(10) 70021-5

Meister P, Towbin BD, Pike BL, Ponti A, Gasser SM. 2010b. The spatial dynamics of tissue-specific promoters during C. elegans development. Genes Dev 24: 766-782. doi:10.1101/gad .559610

Melcon G, Kozlov S, Cutler DA, Sullivan T, Hernandez L, Zhao P, Mitchell S, Nader G, Bakay M, Rottman JN, et al. 2006. Loss of emerin at the nuclear envelope disrupts the Rb1/E2F and MyoD pathways during muscle regeneration. Hum Mol Genet 15: 637-651. doi:10.1093/hmg/ddi479

Mounkes LC, Kozlov SV, Rottman JN, Stewart CL. 2005. Expression of an LMNA-N195K variant of A-type lamins results in cardiac conduction defects and death in mice. Human Mol Genet 14: 2167-2180. doi:10.1093/ hmg/ddi221

Muñoz-Jiménez C, Ayuso C, Dobrzynska A, Torres-Mendéz A, Ruiz PDLC, Askjaer P. 2017. An efficient FLP-based toolkit for spatiotemporal control of gene expression in Caenorhabditis elegans. Genetics 206: 1763-1778. doi:10.1534/genetics .117 .201012

Narasimhan K, Lambert SA, Yang AW, Riddell J, Mnaimneh S, Zheng H, Albu M, Najafabadi HS, Reece-Hoyes JS, Fuxman Bass JI, et al. 2015. Mapping and analysis of Caenorhabditis elegans transcription factor sequence specificities. Elife 4: e06967. doi:10.7554/eLife.06967
Nigg EA. 1992. Assembly and cell cycle dynamics of the nuclear lamina. Semin Cell Biol 3: 245-253. doi:10.1016/1043-4682 (92)90026-R

Osmanagic-Myers S, Dechat T, Foisner R. 2015. Lamins at the crossroads of mechanosignaling. Genes Dev 29: 225-237. doi:10.1101/gad.255968.114

Ozaki T, Saijo M, Murakami K, Enomoto H, Taya Y, Sakiyama S. 1994. Complex formation between lamin A and the retinoblastoma gene product: identification of the domain on lamin A required for its interaction. Oncogene 9: 26492653.

Pekovic V, Harborth J, Broers JLV, Ramaekers FCS, van Engelen B, Lammens M, von Zglinicki T, Foisner R, Hutchison C, Markiewicz E. 2007. Nucleoplasmic LAP2a-lamin A complexes are required to maintain a proliferative state in human fibroblasts. I Cell Biol 176: 163-172. doi:10.1083/jcb .200606139

Perovanovic J, Dell'Orso S, Gnochi VF, Jaiswal JK, Sartorelli V, Vigouroux C, Mamchaoui K, Mouly V, Bonne G, Hoffman EP. 2016. Laminopathies disrupt epigenomic developmental programs and cell fate. Sci Transl Med 8: 335ra58. doi:10 $.1126 /$ scitranslmed.aad4991

Petrella LN, Wang W, Spike CA, Rechtsteiner A, Reinke V, Strome S. 2011. synMuv B proteins antagonize germline fate in the intestine and ensure C. elegans survival. Development 138: 1069-1079. doi:10.1242/dev.059501

Poleshko A, Mansfield KM, Burlingame CC, Andrake MD, Shah NR, Katz RA. 2013. The human protein PRR14 tethers heterochromatin to the nuclear lamina during interphase and mitotic exit. Cell Rep 5: 292-301. doi:10.1016/j.celrep 2013.09.024

Ragoczy T, Bender MA, Telling A, Byron R, Groudine M. 2006. The locus control region is required for association of the murine $\beta$-globin locus with engaged transcription factories during erythroid maturation. Genes Dev 20: 1447-1457. doi:10.1101/ gad.1419506

Reddy KL, Zullo JM, Bertolino E, Singh H. 2008. Transcriptional repression mediated by repositioning of genes to the nuclear lamina. Nature 452: 243-247. doi:10.1038/nature 06727

Sharma R, Ritler D, Meister P. 2016. Tools for DNA adenine methyltransferase identification analysis of nuclear organization during C. elegans development. Genesis 54: 151-159. doi: $10.1002 /$ dvg. 22925

Solovei I, Wang AS, Thanisch K, Schmidt CS, Krebs S, Zwerger M, Cohen TV, Devys D, Foisner R, Peichl L, et al. 2013. LBR and lamin $\mathrm{A} / \mathrm{C}$ sequentially tether peripheral heterochromatin and inversely regulate differentiation. Cell 152: 584-598. doi:10.1016/j.cell.2013.01.009

Stewart CL, Kozlov S, Fong LG, Young SG. 2007. Mouse models of the laminopathies. Exp Cell Res 313: 2144-2156. doi:10 $.1016 /$ j.yexcr.2007.03.026

Strelkov SV, Schumacher J, Burkhard P, Aebi U, Herrmann H. 2004. Crystal structure of the human lamin A coil 2B dimer: implications for the head-to-tail association of nuclear lamins. J Mol Biol 343: 1067-1080. doi:10.1016/j.jmb.2004.08 .093

Timmons L, Court DL, Fire A. 2001. Ingestion of bacterially expressed dsRNAs can produce specific and potent genetic interference in Caenorhabditis elegans. Gene 263: 103-112. doi:10 .1016/S0378-1119(00)00579-5

Towbin BD, González-Aguilera C, Sack R, Gaidatzis D, Kalck V, Meister P, Askjaer P, Gasser SM. 2012. Step-wise methylation of histone $\mathrm{H} 3 \mathrm{~K} 9$ positions heterochromatin at the 
LAD misorganization and its suppression in EDMD

nuclear periphery. Cell 150: 934-947. doi:10.1016/j.cell.2012 .06 .051

Venolia L, Waterston RH. 1990. The unc-45 gene of Caenorhabditis elegans is an essential muscle-affecting gene with maternal expression. Genetics 126: 345-353.

Vlcek S, Foisner R. 2007. A-type lamin networks in light of laminopathic diseases. Biochim Biophys Acta 1773: 661-674. doi:10.1016/j.bbamcr.2006.07.002

Waterston RH, Fishpool RM, Brenner S. 1977. Mutants affecting paramyosin in Caenorhabditis elegans. J Mol Biol 117: 679697. doi:10.1016/0022-2836/77/90064-X
Wiesel N, Mattout A, Melcer S, Melamed-Book N, Herrmann H, Medalia O, Aebi U, Gruenbaum Y. 2008. Laminopathic mutations interfere with the assembly, localization, and dynamics of nuclear lamins. Proc Natl Acad Sci 105: 180-185. doi:10 $.1073 /$ pnas.0708974105

Worman HI, Bonne G. 2007. 'Laminopathies': a wide spectrum of human diseases. Exp Cell Res 313: 2121-2133. doi:10.1016/j .yexcr.2007.03.028

Yang M, Yuan Z-M. 2015. A novel role of PRR14 in the regulation of skeletal myogenesis. Cell Death Dis 6: e1734. doi:10.1038/ cddis. 2015.103 


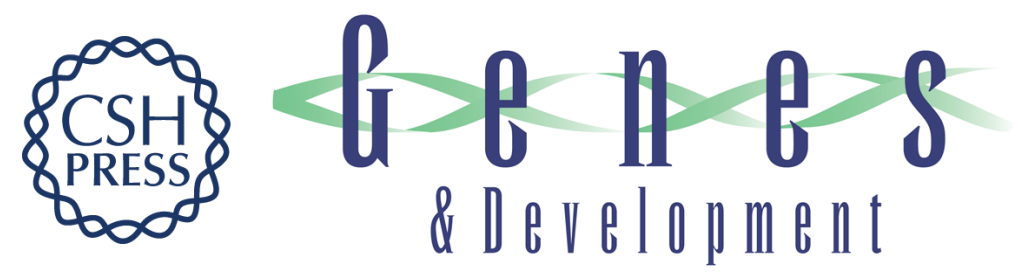

\section{Loss of an H3K9me anchor rescues laminopathy-linked changes in nuclear organization and muscle function in an Emery-Dreifuss muscular dystrophy model}

Jennifer C. Harr, Christoph D. Schmid, Celia Muñoz-Jiménez, et al.

Genes Dev. 2020, 34: originally published online March 5, 2020

Access the most recent version at doi:10.1101/gad.332213.119

Supplemental Material

References

Creative

Commons

License

Email Alerting

Service
http://genesdev.cshlp.org/content/suppl/2020/03/04/gad.332213.119.DC1

This article cites 58 articles, 20 of which can be accessed free at: http://genesdev.cshlp.org/content/34/7-8/560.full.html\#ref-list-1

This article, published in Genes \& Development, is available under a Creative Commons License (Attribution-NonCommercial 4.0 International), as described at http://creativecommons.org/licenses/by-nc/4.0/.

Receive free email alerts when new articles cite this article - sign up in the box at the top right corner of the article or click here.

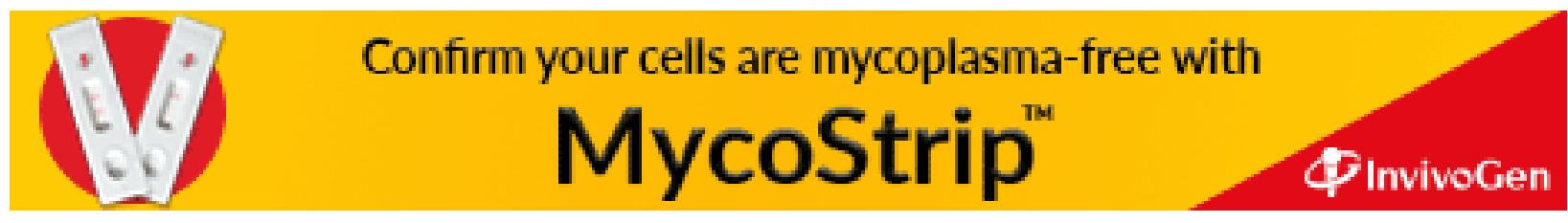

